\title{
OPERATOR ALGEBRAS FOR ANALYTIC VARIETIES
}

\author{
KENNETH R. DAVIDSON, CHRISTOPHER RAMSEY, AND ORR MOSHE SHALIT \\ Dedicated to the memory of William B. Arveson
}

\begin{abstract}
We study the isomorphism problem for the multiplier algebras of irreducible complete Pick kernels. These are precisely the restrictions $\mathcal{M}_{V}$ of the multiplier algebra $\mathcal{M}$ of Drury-Arveson space to a holomorphic subvariety $V$ of the unit ball $\mathbb{B}_{d}$.

We find that $\mathcal{M}_{V}$ is completely isometrically isomorphic to $\mathcal{M}_{W}$ if and only if $W$ is the image of $V$ under a biholomorphic automorphism of the ball. In this case, the isomorphism is unitarily implemented. This is then strengthened to show that when $d<\infty$ every isometric isomorphism is completely isometric.

The problem of characterizing when two such algebras are (algebraically) isomorphic is also studied. When $V$ and $W$ are each a finite union of irreducible varieties and a discrete variety, when $d<\infty$, an isomorphism between $\mathcal{M}_{V}$ and $\mathcal{M}_{W}$ determines a biholomorphism (with multiplier coordinates) between the varieties; and the isomorphism is composition with this function. These maps are automatically weak-* continuous.

We present a number of examples showing that the converse fails in several ways. We discuss several special cases in which the converse does holdparticularly, smooth curves and Blaschke sequences.

We also discuss the norm closed algebras associated to a variety, and point out some of the differences.
\end{abstract}

\section{INTRODUCTION}

In this paper, we study operator algebras of multipliers on reproducing kernel Hilbert spaces associated to analytic varieties in the unit ball of $\mathbb{C}^{d}$. The model is the multiplier algebra $\mathcal{M}_{d}$ of the Drury-Arveson space, a.k.a. the symmetric Fock space. The generators, multiplication by coordinate functions, form a $d$-tuple which is universal for commuting row contractions [8]. The Hilbert space is a reproducing kernel Hilbert space which is a complete Nevanlinna-Pick kernel [15], and in fact when $d=\infty$ is the universal complete NP kernel [1]. For these reasons, this space and its multiplier algebra have received a lot of attention in recent years.

In this paper, we are concerned with multipliers on subspaces of Drury-Arveson space spanned by the kernel functions they contain. By results in [14, these operator algebras are also complete quotients of $\mathcal{M}_{d}$ by WOT-closed ideals. The zero set is always an analytic variety $V$ in the ball, and the multiplier algebra $\mathcal{M}_{V}$ is a space of holomorphic functions on $V$.

Received by the editors January 20, 2012 and, in revised form, January 13, 2013.

2010 Mathematics Subject Classification. Primary 47L30, 47A13, 46E22.

Key words and phrases. Non-selfadjoint operator algebras, reproducing kernel Hilbert spaces.

The first author was partially supported by an NSERC grant.

The third author was supported by ISF Research Grant no. 474/12 and by EU FP7/2007-2013 Grant no. 321749. 
The main question that we address is when two such algebras are isomorphic. We find first that two such algebras $\mathcal{M}_{V}$ and $\mathcal{M}_{W}$ are completely isometrically isomorphic if and only if there is a biholomorphic automorphism of the ball that carries $V$ onto $W$. In this case, the isomorphism is unitarily implemented.

The question of algebraic isomorphism (which implies continuous algebraic isomorphism because the algebras are semisimple) is much more subtle. In an earlier paper [16], the authors considered the case of homogeneous varieties. We showed, under some extra assumptions on the varieties, that the algebras are isomorphic if and only if there is a biholomorphic map of one variety onto the other. In a recent paper, Michael Hartz 20] was able to establish this result in complete generality.

In this paper, we establish a special case of what should be the easy direction, showing that an isomorphism determines a biholomorphism of $V$ onto $W$ when $d<\infty$. This turns out to be rather subtle, and we need to restrict our attention to the case in which the varieties are a finite union of irreducible varieties and a discrete variety. The isomorphism is just composition with this biholomorphism.

These methods also allow us to show that an isometric isomorphism is just composition with a conformal automorphism of the ball, and thus is completely isometric and unitarily implemented.

Some counterexamples show that a biholomorphism between varieties does not always yield an isomorphism of the multiplier algebras. We discuss a number of cases where we can establish the desired converse.

Arias and Latrémolière [5] have an interesting paper in which they study certain operator algebras of this type in the case where the variety is a countable discrete subset of the unit disc which is the orbit of a point under the action of a Fuchsian group. They establish results akin to ours in the completely isometric case using rather different methods.

\section{Reproducing KeRnel Hilbert SPACES ASSOCIATED TO ANALYTIC VARIETIES}

Basic notation. Let $H_{d}^{2}$ be the Drury-Arveson space (see [8]). $H_{d}^{2}$ is the reproducing kernel Hilbert space on $\mathbb{B}_{d}$, the unit ball of $\mathbb{C}^{d}$, with kernel functions

$$
k_{\lambda}(z)=\frac{1}{1-\langle z, \lambda\rangle} \quad \text { for } z, \lambda \in \mathbb{B}_{d} .
$$

We also consider the case $d=\infty$, and then $\mathbb{C}^{d}$ is understood as $\ell^{2}$. We denote by $\mathcal{M}_{d}$ the multiplier algebra $\operatorname{Mult}\left(H_{d}^{2}\right)$ of $H_{d}^{2}$.

Let $Z_{1}, \ldots, Z_{d}$ denote multiplication by the coordinate functions on $H_{d}^{2}$, given by

$$
\left(Z_{i} h\right)(z)=z_{i} h(z) \quad \text { for } i=1, \ldots, d .
$$

Let $\mathcal{A}_{d}$ denote the norm closed algebra generated by $I, Z_{1}, \ldots, Z_{d}$. By [8, Theorem 6.2$], \mathcal{A}_{d}$ is the universal (norm-closed) unital operator algebra generated by a commuting row contraction (see also [24]).

We write $\mathcal{F}=\mathcal{F}(E)$ for the full Fock space

$$
\mathcal{F}=\mathbb{C} \oplus E \oplus(E \otimes E) \oplus(E \otimes E \otimes E) \oplus \ldots,
$$

where $E$ is a $d$-dimensional Hilbert space. Fix an orthonormal basis $\left\{e_{1}, \ldots, e_{d}\right\}$ for $E$. On $\mathcal{F}$, we have the natural shift operators $L_{1}, \ldots, L_{d}$ given by

$$
L_{j} e_{i_{1}} \otimes \cdots \otimes e_{i_{k}}=e_{j} \otimes e_{i_{1}} \otimes \cdots \otimes e_{i_{k}} \quad \text { for } 1 \leq j \leq d .
$$


The non-commutative analytic Toeplitz algebra $\mathcal{L}_{d}$ is defined to be the unital WOTclosed algebra generated by $L_{1}, \ldots, L_{d}$.

When $d$ is understood, we may write $\mathcal{A}, \mathcal{M}, \mathcal{L}$ and $H^{2}$ instead of $\mathcal{A}_{d}, \mathcal{M}_{d}, \mathcal{L}_{d}$ and $H_{d}^{2}$.

The RKHS of a variety. For our purposes, an analytic variety will be understood as the common zero set of a family of $H^{2}$ functions. If $F$ is a subset of $H_{d}^{2}$, considered as functions on $\mathbb{B}_{d}$, let

$$
V(F):=\left\{\lambda \in \mathbb{B}_{d}: f(\lambda)=0 \text { for all } f \in F\right\} .
$$

Propositions 2.1 and 2.2 below both show that there is not much loss of generality in taking this as our definition in this context. In particular, if $f \in \mathcal{M}_{d}$, then $M_{f} 1=f$ is a function in $H_{d}^{2}$. So the zero set of a set of multipliers is an analytic variety.

Define

$$
J_{V}=\{f \in \mathcal{M}: f(\lambda)=0 \text { for all } \lambda \in V\} .
$$

Observe that $J_{V}$ is a WOT-closed ideal in $\mathcal{M}$.

Proposition 2.1. Let $F$ be a subset of $H^{2}$, and let $V=V(F)$. Then

$$
V=V\left(J_{V}\right)=\left\{\lambda \in \mathbb{B}_{d}: f(\lambda)=0 \text { for all } f \in J_{V}\right\} .
$$

Proof. Obviously $V \subseteq V\left(J_{V}\right)$. For the other inclusion, recall that [2, Theorem 9.27] states that a zero set of an $H^{2}$ function is a weak zero set for $\mathcal{M}$ (i.e. the intersection of zero sets of functions in $\mathcal{M})$. Since $V$ is the intersection of zero sets for $H^{2}$, it is a weak zero set for $\mathcal{M}$; i.e., there exists a set $S \subseteq \mathcal{M}$ such that $V=V(S)$. Now, $S \subseteq J_{V}$, so $V=V(S) \supseteq V\left(J_{V}\right)$.

Given the analytic variety $V$, we define a subspace of $H_{d}^{2}$ by

$$
\mathcal{F}_{V}=\overline{\operatorname{span}}\left\{k_{\lambda}: \lambda \in V\right\} .
$$

The Hilbert space $\mathcal{F}_{V}$ is naturally a reproducing kernel Hilbert space of functions on the variety $V$. One could also consider spaces of the form $\mathcal{F}_{S}=\overline{\operatorname{span}}\left\{k_{\lambda}: \lambda \in S\right\}$, where $S$ is an arbitrary subset of the ball. The following proposition shows that there is no loss of generality in considering only analytic varieties generated by $H^{2}$ functions.

Proposition 2.2. Let $S \subseteq \mathbb{B}_{d}$. Let $J_{S}$ denote the set of multipliers vanishing on $S$, and let $I_{S}$ denote the set of all $H^{2}$ functions that vanish on $S$. Then

$$
\mathcal{F}_{S}=\mathcal{F}_{V\left(I_{S}\right)}=\mathcal{F}_{V\left(J_{S}\right)} \text {. }
$$

Proof. Clearly $\mathcal{F}_{S} \subseteq \mathcal{F}_{V\left(I_{S}\right)}$. Let $f \in \mathcal{F}_{S}^{\perp}$. Then $f(x)=0$ for all $x \in S$, so $f \in I_{S}$. Hence by definition, $f(z)=0$ for all $z \in V=V\left(I_{S}\right)$; whence $f \in \mathcal{F}_{V\left(I_{S}\right)}^{\perp}$. Therefore $\mathcal{F}_{S}=\mathcal{F}_{V\left(I_{S}\right)}$. The extension to zero sets of multipliers follows again from [2, Theorem 9.27].

Remarks 2.3. In general, it is not true that $V\left(I_{S}\right)$ is equal to the smallest analytic variety in the classical sense containing $S \subseteq \mathbb{B}_{d}$. In fact, by Weierstrass's Factorization Theorem, every discrete set $Z=\left\{z_{n}\right\}_{n=1}^{\infty}$ in $\mathbb{D}$ is the zero set of some holomorphic function on $\mathbb{D}$. However, if the sequence $Z$ is not a Blaschke sequence, then there is no non-zero function in $H^{2}$ that vanishes on all of it. So here $I_{Z}=\{0\}$, and therefore $V\left(I_{Z}\right)=\mathbb{D}$. 
One very nice property of classical varieties is that the definition is local. Because our functions must be multipliers, a strictly local definition does not seem to be possible. However, one could consider the following variant: $V$ is a variety if for each point $\lambda \in \mathbb{B}_{d}$, there is an $\varepsilon>0$ and a finite set $f_{1}, \ldots, f_{n}$ in $\mathcal{M}_{d}$ so that

$$
b_{\varepsilon}(\lambda) \cap V=\left\{z \in b_{\varepsilon}(\lambda): 0=f_{1}(z)=\cdots=f_{n}(z)\right\} .
$$

We do not know if every variety of this type is actually the intersection of zero sets.

In particular, we will say that a variety $V$ is irreducible if for any regular point $\lambda \in$ $V$, the intersection of zero sets of all multipliers vanishing on a small neighbourhood $V \cap b_{\varepsilon}(\lambda)$ is exactly $V$. However we do not know whether an irreducible variety is connected. A local definition of our varieties would presumably clear up this issue.

Ideals and invariant subspaces. We will apply some results of Davidson-Pitts [14, Theorem 2.1] and [15, Corollary 2.3] to the commutative context.

In the first paper, a bijective correspondence is established between the collection of wOT-closed ideals $J$ of $\mathcal{L}_{d}$ and the complete lattice of subspaces which are invariant for both $\mathcal{L}_{d}$ and its commutant $\mathcal{R}_{d}$, the algebra of right multipliers. The pairing is just the map taking an ideal $J$ to its closed range $\mu(J):=\overline{J \mathcal{F}}$. The inverse map takes a subspace $N$ to the ideal $J$ of elements with range contained in $N$.

In [15. Theorem 2.1], it is shown that the quotient algebra $\mathcal{L}_{d} / J$ is completely isometrically isomorphic and WOT-homeomorphic to the compression of $\mathcal{L}_{d}$ to $\mu(J)^{\perp}$. In particular, [15. Corollary 2.3] shows that the multiplier algebra $\mathcal{M}_{d}$ is completely isometrically isomorphic to $\mathcal{L}_{d} / \mathcal{C}$, where $\mathcal{C}$ is the wOT-closure of the commutator ideal of $\mathcal{L}_{d}$. In particular, $\mu(\mathcal{C})^{\perp}=H_{d}^{2}$.

It is easy to see that there is a bijective correspondence between the lattice of wOT-closed ideals $\operatorname{Id}\left(\mathcal{M}_{d}\right)$ of $\mathcal{M}_{d}$ and the wOT-closed ideals of $\mathcal{L}_{d}$ which contain $\mathcal{C}$. Similarly there is a bijective correspondence between invariant subspaces $N$ of $\mathcal{M}_{d}$ and invariant subspaces of $\mathcal{L}_{d}$ which contain $\mu(\mathcal{C})=H_{d}^{2 \perp}$. Since the algebra $\mathcal{M}_{d}$ is abelian, it is also the quotient of $\mathcal{R}_{d}$ by its commutator ideal, which also has range $H_{d}^{2 \perp}$. So the subspace $N \oplus H_{d}^{2 \perp}$ is invariant for both $\mathcal{L}_{d}$ and $\mathcal{R}_{d}$. Therefore an application of [14, Theorem 2.1] yields the following consequence:

Theorem 2.4. Define the map $\alpha: \operatorname{Id}\left(\mathcal{M}_{d}\right) \rightarrow \operatorname{Lat}\left(\mathcal{M}_{d}\right)$ by $\alpha(J)=\overline{J 1}$. Then $\alpha$ is a complete lattice isomorphism whose inverse $\beta$ is given by

$$
\beta(N)=\left\{f \in \mathcal{M}_{d}: f \cdot 1 \in N\right\} .
$$

Moreover [15, Theorem 2.1] then yields:

Theorem 2.5. If $J$ is a wOT-closed ideal of $\mathcal{M}_{d}$ with range $N$, then $\mathcal{M}_{d} / J$ is completely isometrically isomorphic and WOT-homeomorphic to the compression of $\mathcal{M}_{d}$ to $N^{\perp}$.

The multiplier algebra of a variety. The reproducing kernel Hilbert space $\mathcal{F}_{V}$ comes with its multiplier algebra $\mathcal{M}_{V}=\operatorname{Mult}\left(\mathcal{F}_{V}\right)$. This is the algebra of all functions $f$ on $V$ such that $f h \in \mathcal{F}_{V}$ for all $h \in \mathcal{F}_{V}$. A standard argument shows that each multiplier determines a bounded linear operator $M_{f} \in \mathcal{B}\left(\mathcal{F}_{V}\right)$ given by $M_{f} h=f h$. We will usually identify the function $f$ with its multiplication operator $M_{f}$. We will also identify the subalgebra of $\mathcal{B}\left(\mathcal{F}_{V}\right)$ consisting of the $M_{f}$ 's and the algebra of functions $\mathcal{M}_{V}$ (endowed with the same norm). One reason to distinguish $f$ and $M_{f}$ is that sometimes we need to consider the adjoints of the operators $M_{f}$. 
The distinguishing property of these adjoints is that $M_{f}^{*} k_{\lambda}=\overline{f(\lambda)} k_{\lambda}$ for $\lambda \in V$, in the sense that if $A^{*} k_{\lambda}=\overline{f(\lambda)} k_{\lambda}$ for $\lambda \in V$, then $f$ is a multiplier.

The space $\mathcal{F}_{V}$ is therefore invariant for the adjoints of multipliers, and hence it is the complement of an invariant subspace of $\mathcal{M}$. Thus an application of Theorem 2.5 and the complete Nevanlinna-Pick property yields:

Proposition 2.6. Let $V$ be an analytic variety in $\mathbb{B}_{d}$. Then

$$
\mathcal{M}_{V}=\left\{\left.f\right|_{V}: f \in \mathcal{M}\right\} \text {. }
$$

Moreover, the mapping $\varphi: \mathcal{M} \rightarrow \mathcal{M}_{V}$ given by $\varphi(f)=\left.f\right|_{V}$ induces a completely isometric isomorphism and wOT-homeomorphism of $\mathcal{M} / J_{V}$ onto $\mathcal{M}_{V}$. For any $g \in \mathcal{M}_{V}$ and any $f \in \mathcal{M}$ such that $\left.f\right|_{V}=g$, we have $M_{g}=\left.P_{\mathcal{F}_{V}} M_{f}\right|_{\mathcal{F}_{V}}$. Given any $F \in M_{k}\left(\mathcal{M}_{V}\right)$, one can choose $\widetilde{F} \in M_{k}(\mathcal{M})$ so that $\left.\widetilde{F}\right|_{V}=F$ and $\|\widetilde{F}\|=\|F\|$.

Proof. Theorem 2.5 provides the isomorphism between $\mathcal{M} / J_{V}$ and the restriction of the multipliers to $N^{\perp}$, where $N=\overline{J_{V} 1}$. Since $J_{V}$ vanishes on $V$, if $f \in J_{V}$, we have

$$
\left\langle M_{f} h, k_{\lambda}\right\rangle=\left\langle h, M_{f}^{*} k_{\lambda}\right\rangle=0 \text { for all } \lambda \in V \text { and } h \in H_{d}^{2} .
$$

So $N$ is orthogonal to $\mathcal{F}_{V}$. Conversely, if $M_{f}$ has range orthogonal to $\mathcal{F}_{V}$, the same calculation shows that $f \in J_{V}$. Since the pairing between subspaces and ideals is bijective, we deduce that $N=\mathcal{F}_{V}^{\perp}$. The mapping of $\mathcal{M} / J_{V}$ into $\mathcal{M}_{V}$ is given by compression to $\mathcal{F}_{V}$ by sending $f$ to $\left.P_{\mathcal{F}_{V}} M_{f}\right|_{\mathcal{F}_{V}}$.

It is now evident that the restriction of a multiplier $f$ in $\mathcal{M}$ to $V$ yields a multiplier on $\mathcal{F}_{V}$, and that the norm is just $\left\|f+J_{V}\right\|=\left\|\left.P_{\mathcal{F}_{V}} M_{f}\right|_{\mathcal{F}_{V}}\right\|$. We need to show that this map is surjective and completely isometric. This follows from the complete Nevanlinna-Pick property as in [15. Corollary 2.3]. Indeed, if $F \in$ $M_{k}\left(\mathcal{M}_{V}\right)$ with $\|F\|=1$, then standard computations show that if $\lambda_{1}, \ldots, \lambda_{n}$ lie in $V$, then

$$
\left[\left(I_{k}-F\left(\lambda_{j}\right) F\left(\lambda_{i}\right)^{*}\right)\left\langle k_{\lambda_{i}}, k_{\lambda_{j}}\right\rangle\right]_{n \times n}
$$

is positive semidefinite. By [15], this implies that there is a matrix multiplier $\widetilde{F} \in M_{k}(\mathcal{M})$ with $\|\widetilde{F}\|=1$ such that $\left.\widetilde{F}\right|_{V}=F$.

We can argue as in the previous subsection that there is a bijective correspondence between WOT-closed ideals of $\mathcal{M}_{V}$ and its invariant subspaces:

Corollary 2.7. Define the map $\alpha: \operatorname{Id}\left(\mathcal{M}_{V}\right) \rightarrow \operatorname{Lat}\left(\mathcal{M}_{V}\right)$ by $\alpha(J)=\overline{J 1}$. Then $\alpha$ is a complete lattice isomorphism whose inverse $\beta$ is given by

$$
\beta(N)=\left\{f \in \mathcal{M}_{V}: f \cdot 1 \in N\right\} .
$$

Remark 2.8. By Theorem 4.2 in [1, every irreducible complete Nevanlinna-Pick kernel is equivalent to the restriction of the kernel of Drury-Arveson space to a subset of the ball. It follows from this and from the above discussion that every multiplier algebra of an irreducible complete Nevanlinna-Pick kernel is completely isometrically isomorphic to one of the algebras $\mathcal{M}_{V}$ that we are considering here.

Remark 2.9. By the universality of $Z_{1}, \ldots, Z_{d}[8$, for every unital operator algebra $\mathcal{B}$ that is generated by a pure commuting row contraction $T=\left(T_{1}, \ldots, T_{d}\right)$, there exists a unital homomorphism $\varphi_{T}: \mathcal{M} \rightarrow \mathcal{B}$ that gives rise to a natural functional calculus

$$
f\left(T_{1}, \ldots, T_{d}\right)=\varphi_{T}(f) \quad \text { for } f \in \mathcal{M} .
$$


So it makes sense to say that a commuting row contraction $T$ annihilates $J_{V}$ if $\varphi_{T}$ vanishes on $J_{V}$. By Proposition 2.6 we may identify $\mathcal{M}_{V}$ with the quotient $\mathcal{M} / J_{V}$, thus we may identify $\mathcal{M}_{V}$ as the universal wOT-closed unital operator algebra generated by a pure commuting row contraction $T=\left(T_{1}, \ldots, T_{d}\right)$ that annihilates $J_{V}$.

\section{The Character SPACE of $\mathcal{M}_{V}$}

If $A$ is a Banach algebra, denote the set of multiplicative linear functionals on $A$ by $M(A)$ and endow this space with the weak-* topology. We refer to elements of $M(A)$ as characters. Note that all characters are automatically unital and continuous with norm one. When $A$ is an operator algebra, characters are completely contractive as well.

When $V$ is an analytic variety in $\mathbb{B}_{d}$, we will abuse notation and let $Z_{1}, \ldots, Z_{d}$ also denote the images of the coordinate functions $Z_{1}, \ldots, Z_{d}$ of $\mathcal{M}$ in $\mathcal{M}_{V}$. Since $\left[Z_{1}, \ldots, Z_{d}\right]$ is a row contraction,

$$
\left\|\left(\rho\left(Z_{1}\right), \ldots, \rho\left(Z_{d}\right)\right)\right\| \leq 1 \quad \text { for all } \rho \in M\left(\mathcal{M}_{V}\right) .
$$

The map $\pi: M\left(\mathcal{M}_{V}\right) \rightarrow \overline{\mathbb{B}}_{d}$ given by

$$
\pi(\rho)=\left(\rho\left(Z_{1}\right), \ldots, \rho\left(Z_{d}\right)\right)
$$

is continuous as a map from $M\left(\mathcal{M}_{V}\right)$, with the weak-* topology, into $\overline{\mathbb{B}}_{d}$ (endowed with the weak topology in the case $d=\infty)$. We define

$$
\bar{V}^{\mathcal{M}}=\pi\left(\left(M\left(\mathcal{M}_{V}\right)\right)\right) .
$$

Since $\pi$ is continuous, $\bar{V}^{\mathcal{M}}$ is a (weakly) compact subset of $\overline{\mathbb{B}_{d}}$. For every $\lambda \in$ $\bar{V}^{\mathcal{M}}$, the fiber over $\lambda$ is defined to be the set $\pi^{-1}(\lambda)$ in $M\left(\mathcal{M}_{V}\right)$. We will see below that $V \subseteq \bar{V}^{\mathcal{M}}$, and when $d<\infty$, that the fiber over every $\lambda \in V$ is a singleton.

Every unital homomorphism $\varphi: A \rightarrow B$ between Banach algebras induces a mapping $\varphi^{*}: M(B) \rightarrow M(A)$ by $\varphi^{*} \rho=\rho \circ \varphi$. If $\varphi$ is a continuous isomorphism, then $\varphi^{*}$ is a homeomorphism. We will see below that in many cases a homomorphism $\varphi: \mathcal{M}_{V} \rightarrow \mathcal{M}_{W}$ gives rise to an induced map $\varphi^{*}: M\left(\mathcal{M}_{W}\right) \rightarrow M\left(\mathcal{M}_{V}\right)$ which has additional structure. The most important aspect is that $\varphi^{*}$ restricts to a holomorphic map from $W$ into $V$.

The weak-* continuous characters of $\mathcal{M}_{V}$. In the case of $\mathcal{M}_{d}$, the weak-* continuous characters coincide with the point evaluations at points in the open ball [6, 13 .

$$
\rho_{\lambda}(f)=f(\lambda)=\left\langle f \nu_{\lambda}, \nu_{\lambda}\right\rangle \quad \text { for } \lambda \in \mathbb{B}_{d}
$$

where $\nu_{\lambda}=k_{\lambda} /\left\|k_{\lambda}\right\|$. The fibers over points in the boundary sphere are at least as complicated as the fibers in $M\left(H^{\infty}\right)$ [14, which are known to be extremely large 22].

As a quotient of a dual algebra by a weak-* closed ideal, the algebra $\mathcal{M}_{V}$ inherits a weak-* topology. As an operator algebra concretely represented on a reproducing kernel Hilbert space, $\mathcal{M}_{V}$ also has the weak-operator topology (WOT). In [16, Lemma 11.9] we observed that, as is the case for the free semigroup algebras $\mathcal{L}_{d}$ [13], the weak-operator and weak-* topologies on $\mathcal{M}_{V}$ coincide. The setting there was slightly different, but the proof remains the same. It relies on the observation 7]. that $\mathcal{M}_{V}$ has property $\mathbb{A}_{1}(1)$. 
Lemma 3.1. The weak-* and weak-operator topologies on $\mathcal{M}_{V}$ coincide.

Proposition 3.2. The WOT-continuous characters of $\mathcal{M}_{V}$ can be identified with $V$. If $d<\infty, \bar{V}^{\mathcal{M}} \cap \mathbb{B}_{d}=V$. The restriction of each $f \in \mathcal{M}_{V}$ to $V$ is a bounded holomorphic function.

Proof. As $\mathcal{M}_{V}$ is the multiplier algebra of a reproducing kernel Hilbert space on $V$, it is clear that for each $\lambda \in V$, the evaluation functional

$$
\rho_{\lambda}(f)=f(\lambda)=\left\langle f \nu_{\lambda}, \nu_{\lambda}\right\rangle
$$

is a WOT-continuous character.

On the other hand, the quotient map from the free semigroup algebra $\mathcal{L}$ onto $\mathcal{M}_{V}$ is weak-operator continuous. Thus, if $\rho$ is a wOT-continuous character of $\mathcal{M}_{V}$, then it induces a WOT-continuous character on $\mathcal{L}$ by composition. Therefore, using [14, Theorem 2.3], we find that $\rho$ must be equal to the evaluation functional $\rho_{\lambda}$ at some point $\lambda \in \mathbb{B}_{d}$. Moreover, $\rho_{\lambda}$ annihilates $J_{V}$. By Proposition 2.1, the point $\lambda$ lies in $V$.

Assume $d<\infty$. If $\rho$ is a character on $\mathcal{M}_{V}$ such that $\pi(\rho)=\lambda \in \mathbb{B}_{d}$, then again it induces a character $\tilde{\rho}$ on $\mathcal{L}$ with the property that $\tilde{\rho}\left(L_{1}, \ldots, L_{d}\right)=\lambda$. By [14. Theorem 3.3], it follows that $\tilde{\rho}$ is WOT-continuous and coincides with point evaluation. Hence by the previous paragraph, $\lambda$ belongs to $V$. So $\bar{V}^{\mathcal{M}} \cap \mathbb{B}_{d}=V$.

Therefore $\pi: \pi^{-1}(V) \rightarrow V$ is seen to be a homeomorphism between $\pi^{-1}(V)$ endowed with the weak-* topology and $V$ with the (weak) topology induced from $\mathbb{B}_{d}$.

By Proposition 2.6, $\mathcal{M}_{V}$ is a quotient of $\mathcal{M}$, and the map is given by restriction to $V$. Hence $f$ is a bounded holomorphic function by [14, Theorem 3.3] or [8, Proposition 2.2].

Remark 3.3. When $d=\infty$, it may happen that $\pi^{-1}(\lambda)$ has more than one element, and it may also happen that $\bar{V}^{\mathcal{M}} \cap \mathbb{B}_{d} \supsetneq V$. Such examples were found by Michael Hartz (private communication).

\section{Completely isometric isomorphisms}

The completely isometric automorphisms of $\mathcal{M}$ arise as composition with an automorphism of the ball (i.e., a biholomorphism of the ball onto itself). This can be deduced from [14, Section 4], or alternatively from Theorems 3.5 and 3.10 in [26]. In [16, Section 9], we wrote the explicit form of the unitaries on $H^{2}$ that implement these automorphisms. We will use these unitaries to construct unitarily implemented, completely isometric isomorphisms of the multiplier algebras that we are studying. In addition, we will show that all completely isometric isomorphisms of these algebras arise in this way.

Proposition 4.1. Let $V$ and $W$ be varieties in $\mathbb{B}_{d}$. Let $F$ be an automorphism of $\mathbb{B}_{d}$ that maps $W$ onto $V$. Then $f \mapsto f \circ F$ is a unitarily implemented completely isometric isomorphism of $\mathcal{M}_{V}$ onto $\mathcal{M}_{W}$; i.e. $M_{f \circ F}=U M_{f} U^{*}$. The unitary $U^{*}$ is the linear extension of the map

$$
U^{*} k_{w}=c_{w} k_{F(w)} \quad \text { for } w \in W,
$$

where $c_{w}=\left(1-\left\|F^{-1}(0)\right\|^{2}\right)^{1 / 2} \overline{k_{F^{-1}(0)}(w)}$. 
Proof. Let $F$ be such an automorphism, and set $\alpha=F^{-1}(0)$. By [16, Theorem 9.2], the unitary map $U \in \mathcal{B}\left(H^{2}\right)$ is given by

$$
U h=\left(1-\|\alpha\|^{2}\right)^{1 / 2} k_{\alpha}(h \circ F) \quad \text { for } h \in H^{2} .
$$

As $F(W)=V, U$ takes the functions in $H^{2}$ that vanish on $V$ to the functions in $H^{2}$ that vanish on $W$. Therefore it takes $\mathcal{F}_{V}$ onto $\mathcal{F}_{W}$.

Let us compute $U^{*}$. For $h \in H^{2}$ and $w \in W$, we have

$$
\begin{aligned}
\left\langle h, U^{*} k_{w}\right\rangle & =\left\langle U h, k_{w}\right\rangle \\
& =\left\langle\left(1-\|\alpha\|^{2}\right)^{1 / 2} k_{\alpha}(h \circ F), k_{w}\right\rangle \\
& =\left(1-\|\alpha\|^{2}\right)^{1 / 2} k_{\alpha}(w) h(F(w)) \\
& =\left\langle h, c_{w} k_{F(w)}\right\rangle,
\end{aligned}
$$

where $c_{w}=\left(1-\left\|F^{-1}(0)\right\|^{2}\right)^{1 / 2} \overline{k_{F^{-1}(0)}(w)}$. Thus $U^{*} k_{w}=c_{w} k_{F(w)}$. Note that since $U^{*}$ is a unitary, $\left|c_{w}\right|=\left\|k_{w}\right\| /\left\|k_{F(w)}\right\|$.

Finally, we show that conjugation by $U$ implements the isomorphism between $\mathcal{M}_{V}$ and $\mathcal{M}_{W}$ given by composition with $F$. Observe that $U c_{w} k_{F(w)}=k_{w}$. For $f \in \mathcal{M}_{V}$ and $w \in W$,

$$
U M_{f}^{*} U^{*} k_{w}=U M_{f}^{*} c_{w} k_{F(w)}=\overline{f(F(w))} U c_{w} k_{F(w))}=\overline{(f \circ F)(w)} k_{w} .
$$

Therefore $f \circ F$ is a multiplier on $\mathcal{F}_{W}$ and $M_{f \circ F}=U M_{f} U^{*}$.

Now we turn to the converse.

Lemma 4.2. Let $V \subseteq \mathbb{B}_{d}$ and $W \subseteq \mathbb{B}_{d^{\prime}}$ be varieties. Let $\varphi$ be a unital, completely contractive algebra isometric isomorphism of $\mathcal{M}_{V}$ into $\mathcal{M}_{W}$. Then there exists a holomorphic map $F: \mathbb{B}_{d^{\prime}} \rightarrow \mathbb{B}_{d}$ such that

(1) $F(W) \subseteq V$,

(2) $\left.F\right|_{W}=\left.\varphi^{*}\right|_{W}$,

(3) the components $f_{1}, \ldots, f_{d}$ of $F$ form a row contraction of operators in $\mathcal{M}_{d^{\prime}}$,

(4) $\varphi$ is given by composition with $F$, that is,

$$
\varphi(f)=f \circ F \quad \text { for } f \in \mathcal{M}_{V} .
$$

Proof. Consider the image of the coordinate functions $Z_{i}$ in $\mathcal{M}_{V}$. As $\varphi$ is completely contractive, Proposition 2.6 shows that $\left[\varphi\left(Z_{1}\right) \ldots \varphi\left(Z_{d}\right)\right]$ is the restriction to $W$ of a row contractive multiplier $F=\left[f_{1}, \ldots, f_{d}\right]$ with coefficients in $\mathcal{M}_{d^{\prime}}$. As $F$ is contractive as a multiplier, it is also contractive in the sup norm. Moreover, since $\varphi$ is injective, the $f_{i}$ and $F$ are non-constant holomorphic functions. Therefore $F$ must have range in the open ball $\mathbb{B}_{d}$.

Fix $\lambda \in W$, and let $\rho_{\lambda}$ be the evaluation functional at $\lambda$ on $\mathcal{M}_{W}$. Then $\varphi^{*}\left(\rho_{\lambda}\right)$ is a character in $M\left(\mathcal{M}_{V}\right)$. We want to show that it is also an evaluation functional. Compute

$$
\left[\varphi^{*}\left(\rho_{\lambda}\right)\right]\left(Z_{i}\right)=Z_{i}\left(\varphi^{*}\left(\rho_{\lambda}\right)\right)=\rho_{\lambda}\left(\varphi\left(Z_{i}\right)\right)=\varphi\left(Z_{i}\right)(\lambda) .
$$

So $\varphi^{*}\left(\rho_{\lambda}\right)$ lies in the fiber over $\left(\varphi\left(Z_{1}\right)(\lambda), \ldots, \varphi\left(Z_{d}\right)(\lambda)\right)=F(\lambda)$. This is in the interior of the ball. By Proposition [3.2 $\varphi^{*}\left(\rho_{\lambda}\right)$ is the point evaluation functional $\rho_{F(\lambda)}$ and $F(\lambda) \in V$. We abuse notation by saying that $\varphi^{*}\left(\rho_{\lambda}\right) \in V$. 
Finally, for every $f \in \mathcal{M}_{V}$ and every $\lambda \in W$,

$$
\begin{aligned}
\varphi(f)(\lambda) & =\rho_{\lambda}(\varphi(f))=\varphi^{*}\left(\rho_{\lambda}\right)(f) \\
& =\rho_{F(\lambda)}(f)=(f \circ F)(\lambda) .
\end{aligned}
$$

Therefore $\varphi(f)=f \circ F$.

Lemma 4.3. Let $0 \in V \subseteq \mathbb{B}_{d}$ and $0 \in W \subseteq \mathbb{B}_{d^{\prime}}$ be varieties. Let $\varphi: \mathcal{M}_{V} \rightarrow \mathcal{M}_{W}$ be a completely isometric isomorphism such that $\varphi^{*} \rho_{0}=\rho_{0}$. Then there exists an isometric linear map $F$ of $\mathbb{B}_{d^{\prime}} \cap \operatorname{span} W$ onto $\mathbb{B}_{d} \cap \operatorname{span} V$ such that $F(W)=V$, $F(0)=0$ and $\left.F\right|_{W}=\varphi^{*}$.

Proof. By making $d$ smaller, we may assume that $\mathbb{C}^{d}=\operatorname{span} V$. Similarly, we may assume $\mathbb{C}^{d^{\prime}}=\operatorname{span} W$.

By Lemma 4.2 applied to $\varphi$, there is a holomorphic map $F$ of $\mathbb{B}_{d^{\prime}}$ into $\mathbb{B}_{d}$ that implements $\varphi^{*}$. Thus $F(W) \subseteq V$ and $F(0)=0$. By the same lemma applied to $\varphi^{-1}$, there is a holomorphic map $G$ of $\mathbb{B}_{d}$ into $\mathbb{B}_{d^{\prime}}$ that implements $\left(\varphi^{-1}\right)^{*}$. Hence $G(V) \subseteq W$ and $G(0)=0$. Now, $\varphi^{*}$ and $\left(\varphi^{-1}\right)^{*}$ are inverses of each other. Therefore $\left.F \circ G\right|_{V}$ and $\left.G \circ F\right|_{W}$ are the identity maps.

Let $H=F \circ G$. Then $H$ is a holomorphic map of $\mathbb{B}_{d}$ into itself such that $\left.H\right|_{V}$ is the identity. In particular, $H(0)=0$. By [27, Theorem 8.2.2], the fixed point set of $H$ is an affine set equal to the fixed point set of $H^{\prime}(0)$ in $\mathbb{B}_{d}$. Therefore $H$ is the identity on $\mathbb{B}_{d}$ since $\mathbb{C}^{d}=\operatorname{span} V$. Applying the same reasoning to $G \circ F$, we see that $F$ is a biholomorphism of $\mathbb{B}_{d^{\prime}}$ onto $\mathbb{B}_{d}$ such that $F(W)=V$. In particular, $d^{\prime}=d$. It now follows from a theorem of Cartan [27, Theorem 2.1.3] that $F$ is a unitary linear map.

Now we combine these lemmas to obtain the main result of this section.

If $V \subseteq \mathbb{B}_{d}$ and $W \subseteq \mathbb{B}_{d^{\prime}}$ are varieties, then we can consider them both as varieties in $\mathbb{B}_{\max \left(d, d^{\prime}\right)}$. Therefore, we may assume that $d=d^{\prime}$. This does not change the operator algebras. See [16, Remark 8.1].

Theorem 4.4. Let $V$ and $W$ be varieties in $\mathbb{B}_{d}$. Then $\mathcal{M}_{V}$ is completely isometrically isomorphic to $\mathcal{M}_{W}$ if and only if there exists an automorphism $F$ of $\mathbb{B}_{d}$ such that $F(W)=V$.

In fact, every completely isometric isomorphism $\varphi: \mathcal{M}_{V} \rightarrow \mathcal{M}_{W}$ arises as composition $\varphi(f)=f \circ F$, where $F$ is such an automorphism. In this case, $\varphi$ is unitarily implemented by the unitary sending the kernel function $k_{w} \in \mathcal{F}_{W}$ to a scalar multiple of the kernel function $k_{F(w)} \in \mathcal{F}_{V}$.

Proof. If there is such an automorphism, then the two algebras are completely isometrically isomorphic by Proposition 4.1, and the unitary is given explicitly there.

Conversely, assume that $\varphi$ is a completely isometric isomorphism of $\mathcal{M}_{V}$ onto $\mathcal{M}_{W}$. By Lemma 4.2, $\varphi^{*}$ maps $W$ into $V$. Pick a point $w_{0} \in W$ and set $v_{0}=\varphi^{*}\left(w_{0}\right)$. By applying automorphisms of $\mathbb{B}_{d}$ that move $v_{0}$ and $w_{0}$ to 0 respectively, and applying Proposition 4.1, we may assume that $0 \in V$ and $0 \in W$ and $\varphi^{*}(0)=0$.

Now we apply Lemma 4.3 to obtain an isometric linear map $F$ of the ball $\mathbb{B}_{d} \cap$ span $W$ onto the ball $\mathbb{B}_{d} \cap \operatorname{span} V$ such that $\left.F\right|_{W}=\varphi^{*}$. In particular, span $W$ and span $V$ have the same dimension. (Caveat: this is only true in the case that both $V$ and $W$ contain 0 .) We may extend the definition of $F$ to a unitary map on $\mathbb{C}^{d}$, and so it extends to a biholomorphism of $\mathbb{B}_{d}$. 
Now Proposition 4.1 yields a unitary which implements composition by $\varphi^{*}$. By Lemma 4.2, every completely isometric isomorphism $\varphi$ is given as a composition by $\varphi^{*}$. So all maps have the form described.

There is a converse to Lemma 4.2, which may provide an alternative proof for one half of Theorem 4.4. Arguments like the following are not uncommon in the theory of RKHS; see for example [23, Theorem 5].

Proposition 4.5. Let $V \subseteq \mathbb{B}_{d}$ and $W \subseteq \mathbb{B}_{d^{\prime}}$ be varieties. Suppose that there exists a holomorphic map $F: \mathbb{B}_{d^{\prime}} \rightarrow \mathbb{B}_{d}$ that satisfies $F(W) \subseteq V$ such that the components $f_{1}, \ldots, f_{d}$ of $F$ form a row contraction of operators in $\mathcal{M}_{d^{\prime}}$. Then the map given by composition with $F$,

$$
\varphi(f)=f \circ F \quad \text { for } f \in \mathcal{M}_{V},
$$

yields a unital, completely contractive algebra homomorphism of $\mathcal{M}_{V}$ into $\mathcal{M}_{W}$.

Proof. Composition obviously gives rise to a unital homomorphism, so all we have to demonstrate is that $\varphi$ is completely contractive. We make use of the complete NP property of these kernels.

Let $G \in M_{k}\left(\mathcal{M}_{V}\right)$ with $\|G\| \leq 1$. Then for any $N$ points $w_{1}, \ldots, w_{N}$ in $W$, we get $N$ points $F\left(w_{1}\right), \ldots, F\left(w_{N}\right)$ in $V$. The fact that $\|G\| \leq 1$ implies that the $N \times N$ matrix with $k \times k$ matrix entries

$$
\left[\frac{I_{k}-(G \circ F)\left(w_{i}\right)(G \circ F)\left(w_{j}\right)^{*}}{1-\left\langle F\left(w_{i}\right), F\left(w_{j}\right)\right\rangle}\right]_{N \times N} \geq 0 .
$$

Also, since $\|F\| \leq 1$ as a multiplier on $\mathcal{F}_{W}$, we have that

$$
\left[\frac{1-\left\langle F\left(w_{i}\right), F\left(w_{j}\right)\right\rangle}{1-\left\langle w_{i}, w_{j}\right\rangle}\right]_{N \times N} \geq 0 .
$$

Therefore the Schur product of these two positive matrices is positive:

$$
\left[\frac{I_{k}-(G \circ F)\left(w_{i}\right)(G \circ F)\left(w_{j}\right)^{*}}{1-\left\langle w_{i}, w_{j}\right\rangle}\right]_{N \times N} \geq 0 .
$$

Now the complete NP property yields that $G \circ F$ is a contractive multiplier in $M_{k}\left(\mathcal{M}_{W}\right)$.

\section{ISOMORPHISMS OF ALGEBRAS AND BIHOLOMORPHISMS}

We turn now to the question: when does there exist an (algebraic) isomorphism between $\mathcal{M}_{V}$ and $\mathcal{M}_{W}$ ? This problem is more subtle, and we frequently need to assume that the variety sits inside a finite-dimensional ambient space. Even the construction of the biholomorphism seems to rely on some delicate facts about complex varieties.

We begin with a well-known automatic continuity result. Recall that a commutative Banach algebra is semisimple if the Gelfand transform is injective.

Lemma 5.1. Let $V$ and $W$ be varieties in $\mathbb{B}_{d}$. Every homomorphism from $\mathcal{M}_{V}$ to $\mathcal{M}_{W}$ is norm continuous.

Proof. The algebras that we are considering are easily seen to be semisimple. A general result in the theory of commutative Banach algebras says that every homomorphism into a semisimple algebra is automatically continuous (see [11, Prop. 4.2]). 
Lemma 5.2. Let $V$ and $W$ be varieties in $\mathbb{B}_{d}$ and $\mathbb{B}_{d^{\prime}}$, respectively, with $d^{\prime}<\infty$. Let $\varphi: \mathcal{M}_{V} \rightarrow \mathcal{M}_{W}$ be an algebra isomorphism. Suppose that $\lambda$ is an isolated point in $W$. Then $\varphi^{*}\left(\rho_{\lambda}\right)$ is an evaluation functional at a point in $V$.

Proof. The character $\rho_{\lambda}$ is an isolated point in $M\left(\mathcal{M}_{W}\right)$. (Here is where we need $d^{\prime}<\infty$.) Since $\varphi^{*}$ is a homeomorphism, $\varphi^{*}\left(\rho_{\lambda}\right)$ must also be an isolated point in $M\left(\mathcal{M}_{V}\right)$. By Shilov's idempotent theorem (see [9, Theorem 21.5]), the characteristic function $\chi_{\varphi^{*}\left(\rho_{\lambda}\right)}$ of $\varphi^{*}\left(\rho_{\lambda}\right)$ belongs to $\mathcal{M}_{V}$. Now suppose that $\varphi^{*}\left(\rho_{\lambda}\right)$ is in the corona $M\left(\mathcal{M}_{V}\right) \backslash V$. Then $\chi_{\varphi^{*}\left(\rho_{\lambda}\right)}$ vanishes on $V$. Therefore, as an element of a multiplier algebra, this means that $\chi_{\varphi^{*}\left(\rho_{\lambda}\right)}=0$. Therefore $\chi_{\varphi^{*}\left(\rho_{\lambda}\right)}$ must vanish on the entire maximal ideal space, which is a contradiction. Thus $\varphi^{*}\left(\rho_{\lambda}\right)$ lies in $V$.

Next we want to show that any algebra isomorphism $\varphi$ between $\mathcal{M}_{V}$ and $\mathcal{M}_{W}$ must induce a biholomorphism between $W$ and $V$. This identification will be the restriction of $\varphi^{*}$ to the characters of evaluation at points of $W$. In order to achieve this, we need to make some additional assumptions.

Our difficulty is basically that we do not have enough information about varieties. In the classical case, if one takes a regular point $\lambda \in V$, takes the connected component of $\lambda$ in the set of all regular points of $V$, and closes it up (in $\mathbb{B}_{d}$ ), then one obtains a subvariety. Moreover, the closure of the complement of this component is also a variety [28, ch.3, Theorem $1 \mathrm{G}]$.

However, our varieties are the intersections of zero sets of a family of multipliers. Let us say that a variety $V$ is irreducible if for any regular point $\lambda \in V$, the intersection of zero sets of all multipliers vanishing on a small neighbourhood $V \cap$ $b_{\varepsilon}(\lambda)$ is exactly $V$. We do not know, for example, whether an irreducible variety in our sense is connected, nor do we know that if we take an irreducible subvariety of a variety, then there is a complementary subvariety as in the classical case.

A variety $V$ is said to be discrete if it has no accumulation points in $\mathbb{B}_{d}$.

We will resolve this in two situations. The first is the case of a finite union of irreducible varieties and a discrete variety. The second is the case of an isometric isomorphism. In the latter case, the isomorphism will turn out to be completely isometric. This yields a different approach to the results of the previous section. We require $d<\infty$.

We need some information about the maximal ideal space $M\left(\mathcal{M}_{V}\right)$. Recall that there is a canonical projection $\pi$ into $\overline{\mathbb{B}}_{d}$ obtained by evaluation at $\left[Z_{1}, \ldots, Z_{d}\right]$. For any point $\mu$ in the unit sphere, $\pi^{-1}(\mu)$ is the fiber of $M\left(\mathcal{M}_{V}\right)$ over $\mu$. We saw in Proposition 3.2 that for $\lambda \in \mathbb{B}_{d}, d<\infty, \pi^{-1}(\lambda)$ is the singleton $\left\{\rho_{\lambda}\right\}$. The following lemma is analogous to results about Gleason parts for function algebras. However, part (2) shows that this is different from Gleason parts, as disjoint subvarieties of $V$ will be at a distance of less than 2 apart. This is because $\mathcal{M}_{V}$ is a (complete) quotient of $\mathcal{M}_{d}$, and thus the difference $\left\|\rho_{\lambda}-\rho_{\mu}\right\|$ is the same whether evaluated as functionals on $\mathcal{M}_{V}$ or $\mathcal{M}_{d}$. In the latter algebra, $\lambda$ and $\nu$ do lie in the same Gleason part.

Lemma 5.3. Let $V$ be a variety in $\mathbb{B}_{d}$.

(1) Let $\varphi \in \pi^{-1}(\mu)$ for $\mu \in \partial \mathbb{B}_{d}$. Suppose that $\psi \in M\left(\mathcal{M}_{V}\right)$ satisfies $\|\psi-\varphi\|<$ 2. Then $\psi$ also belongs to $\pi^{-1}(\mu)$.

(2) If $\lambda$ and $\mu$ belong to $V$, then $\left\|\rho_{\mu}-\rho_{\lambda}\right\| \leq 2 r<2$, where $r$ is the pseudohyperbolic distance between $\mu$ and $\lambda$. 
Proof. If $\psi \in \pi^{-1}(\nu)$ for $\nu \neq \mu$ in the sphere, then there is an automorphism of $\mathbb{B}_{d}$ that takes $\mu$ to $(1,0, \ldots, 0)$ and $\nu$ to $(-1,0, \ldots, 0)$. Proposition 4.1 shows that composition by this automorphism is a completely isometric automorphism. So we may suppose that $\mu=(1,0, \ldots, 0)$ and $\nu=(-1,0, \ldots, 0)$. But then

$$
\|\psi-\varphi\| \geq\left|(\psi-\varphi)\left(Z_{1}\right)\right|=2 .
$$

Similarly, if $\psi=\rho_{\lambda}$ for some $\lambda \in V$, then for any $0<\varepsilon<1$, there is an automorphism of $\mathbb{B}_{d}$ that takes $\mu$ to $(1,0, \ldots, 0)$ and $\nu$ to $(-1+\varepsilon, 0, \ldots, 0)$. The same conclusion is reached by letting $\varepsilon$ decrease to 0 .

If $\lambda$ and $\mu$ belong to $V$, then there is an automorphism $\gamma$ of $\mathbb{B}_{d}$ sending $\lambda$ to 0 and $\mu$ to some $v:=(r, 0, \ldots, 0)$, where $0<r<1$ is the pseudohyperbolic distance between $\lambda$ and $\mu$. Given any multiplier $f \in \mathcal{M}_{V}$ with $\|f\|=1$, Proposition 2.6 provides a multiplier $\tilde{f}$ in $\mathcal{M}_{d}$ so that $\left.\tilde{f}\right|_{V}=f$ and $\|\tilde{f}\|=1$. In particular, $\tilde{f} \circ \gamma^{-1}$ is holomorphic on $\mathbb{B}_{d}$ and $\left\|\tilde{f} \circ \gamma^{-1}\right\|_{\infty} \leq 1$. Hence the Schwarz Lemma [27, Theorem 8.1.4] shows that

$$
\left|\frac{f(\mu)-f(\lambda)}{1-f(\mu) \overline{f(\lambda)}}\right|=\left|\frac{\tilde{f} \circ \gamma^{-1}(v)-\tilde{f} \circ \gamma^{-1}(0)}{1-\tilde{f} \circ \gamma^{-1}(v) \tilde{f} \circ \gamma^{-1}(0)}\right| \leq r .
$$

Hence

$$
\left\|\rho_{\mu}-\rho_{\lambda}\right\|=\sup _{\|f\| \leq 1}\left|\left(\rho_{\mu}-\rho_{\lambda}\right)(f)\right| \leq r \sup _{\|f\| \leq 1}|1-f(\mu) \overline{f(\lambda)}| \leq 2 r .
$$

This provides some immediate information about norm continuous maps between these maximal ideal spaces.

Corollary 5.4. Assume that $d<\infty$. Suppose that $\varphi$ is a continuous algebra homomorphism of $\mathcal{M}_{V}$ into $\mathcal{M}_{W}$.

(1) Then $\varphi^{*}$ maps each irreducible subvariety of $W$ into $V$ or into a single fiber of the corona.

(2) If $\varphi$ is an isomorphism, and $V$ and $W$ are the disjoint union of finitely many irreducible subvarieties, then $\varphi^{*}$ must map $W$ onto $V$.

(3) If $\varphi$ is an isometric isomorphism, then $\varphi^{*}$ maps $W$ onto $V$ and preserves the pseudohyperbolic distance.

Proof. (1) Let $W_{1}$ be an irreducible subvariety of $W$, and let $\lambda$ be any regular point of $W_{1}$. We do not assert that $W_{1}$ is connected.

Suppose that $\varphi^{*}\left(\rho_{\lambda}\right)$ is a point evaluation at some point $\mu$ in $\mathbb{B}_{d}$. Then by Proposition 3.2, $\mu$ belongs to $V$. Since $\varphi$ is norm continuous, by Lemma 5.3 it must map the connected component of $\lambda$ into a connected component of $V$.

Similarly, suppose that $\varphi^{*}$ maps $\rho_{\lambda}$ into a fiber of the corona. Without loss of generality, we may suppose that it is the fiber over $(1,0, \ldots, 0)$. Since $\varphi$ is norm continuous, by Lemma 5.3 it must map the connected component of $\lambda$ into this fiber as well. Suppose that there is some point $\mu$ in $W_{1}$ mapped into $V$ or into another fiber. So the whole connected component of $\mu$ is also mapped into $V$ or another fiber. Then the function $h=\varphi\left(Z_{1}\right)-1$ vanishes on the component of $\lambda$ but does not vanish on the component containing $\mu$. This contradicts the fact that $W_{1}$ is irreducible. Thus the whole subvariety must map entirely into a single fiber or entirely into $V$.

(2) Suppose that $W$ is the union of irreducible subvarieties $W_{1}, \ldots, W_{n}$. Fix a point $\lambda \in W_{1}$. For each $2 \leq i \leq n$, there is a multiplier $h_{i} \in \mathcal{M}_{d^{\prime}}$ which vanishes 
on $W_{i}$ but $h_{i}(\lambda) \neq 0$. Hence $h=\left.h_{2} h_{3} \cdots h_{k}\right|_{W}$ belongs to $\mathcal{M}_{W}$ and vanishes on $\bigcup_{i=2}^{k} W_{i}$ but not on $W_{1}$. Therefore $\varphi^{-1}(h)=f$ is a non-zero element of $\mathcal{M}_{V}$. Suppose that $\varphi^{*}\left(W_{1}\right)$ is contained in a fiber over a point in the boundary of the sphere, say $(1,0, \ldots, 0)$. Since $Z_{1}-1$ is non-zero on $V$, we see that $\left(Z_{1}-1\right) f$ is not the zero function. However, $\left(Z_{1}-1\right) f$ vanishes on $\varphi^{*}\left(W_{1}\right)$. Therefore $\varphi\left(\left(Z_{1}-1\right) f\right)$ vanishes on $W_{1}$ and on $\bigcup_{i=2}^{k} W_{i}$. Hence $\varphi\left(\left(Z_{1}-1\right) f\right)=0$, contradicting injectivity. We deduce that $W_{1}$ is mapped into $V$. $V$.

By interchanging the roles of $V$ and $W$, we deduce that $\varphi^{*}$ must map $W$ onto

(3) In the isometric case, we can make use of Lemma 5.3 (2) because then $\varphi^{*}$ is also isometric. Therefore all of $W$ is mapped by $\varphi^{*}$ either into $V$ or into a single fiber. In the latter case, we may suppose that the fiber is over $(1,0, \ldots, 0)$. Then $\varphi\left(Z_{1}-1\right)$ will vanish on all of $W$, and hence $\varphi\left(Z_{1}-1\right)=0$, contradicting injectivity. Thus $W$ is mapped into $V$. Reversing the role of $V$ and $W$ shows that this map is also onto $V$.

The proof of Lemma 5.3 (2) actually yields more information, namely that $\left\|\rho_{\lambda}-\rho_{\mu}\right\|$ is a function of the pseudohyperbolic distance $r$,

$$
\left\|\rho_{\lambda}-\rho_{\mu}\right\|=r \sup _{\|f\| \leq 1}|1-f(\mu) \overline{f(\lambda)}|
$$

In the proof of that lemma we only used the fact that the left hand side is less than or equal to the right hand side, but it is easy to see that one obtains equality by choosing a particular $f$. So the fact that the quantities $\left\|\rho_{\lambda}-\rho_{\mu}\right\|$ and $\sup _{\|f\| \leq 1}|1-f(\mu) \overline{f(\lambda)}|$ are preserved by an isometric isomorphism implies that the pseudohyperbolic distance $r$ is also preserved.

Remarks 5.5. (1) In a previous version of this paper, we claimed incorrectly that if $\varphi$ is a surjective continuous homomorphism of $\mathcal{M}_{V}$ onto $\mathcal{M}_{W}$, then $\varphi^{*}$ must map $W$ into $V$. This is false, and we thank Michael Hartz for pointing this out. This follows from Hoffman's theory [21] of analytic disks in the corona of $H^{\infty}$. There is an analytic map $L$ of the unit disk $\mathbb{D}$ into the corona of $M\left(H^{\infty}\right)$, mapping onto a Gleason part, with the property that $\varphi(h)(z)=h(L(z))$ is a homomorphism of $H^{\infty}$ onto itself [17, ch. X, $\S 1$ ]. Therefore the map $\varphi^{*}$ maps the disk into the corona via $L$.

(2) The main obstacle preventing us from establishing part (2) of the corollary in greater generality is that we do not know that if $\lambda \in W$, then there is an irreducible subvariety $W_{1} \subset W$ containing $\lambda$ and another subvariety $W_{2} \subset W$ so that $\lambda \notin W_{2}$ and $W=W_{1} \cup W_{2}$. As mentioned in the introduction, for any classical analytic variety this is possible [28, ch. 3, Theorem 1G]. But our definition requires these subvarieties to be the intersection of zero sets of multipliers. Moreover, our proof makes significant use of these functions. So we cannot just redefine our varieties to have a local definition as in the classical case, even if we impose the restriction that all functions are multipliers. A better understanding of varieties in our context is needed.

(3) Costea, Sawyer and Wick [10] establish a corona theorem for the algebra $\mathcal{M}_{d}, d<\infty$. That is, the closure of the ball $\mathbb{B}_{d}$ in $M\left(\mathcal{M}_{d}\right)$ is the entire maximal ideal space. This result may also hold for the quotients $\mathcal{M}_{V}$, but we are not aware of any direct proof deducing this from the result for the whole ball. 
A corona theorem for $\mathcal{M}_{V}$ would resolve the difficulties in case (2). The topology on $V=\mathbb{B}_{d} \cap M\left(\mathcal{M}_{V}\right)$ coincides with the usual one. In particular, each component has closed complement. The corona theorem would establish that every open subset of any fiber is in the closure of its complement. Thus any homeomorphism $\varphi^{*}$ of $M\left(\mathcal{M}_{W}\right)$ onto $M\left(\mathcal{M}_{V}\right)$ must take $W$ onto $V$. However, it is likely that the corona theorem for $\mathcal{M}_{V}$ is much more difficult than our problem.

Now we can deal with the case in which our variety is a finite union of nice subvarieties, where nice will mean either irreducible or discrete.

Theorem 5.6. Let $V$ and $W$ be varieties in $\mathbb{B}_{d}$, with $d<\infty$, which are the union of finitely many irreducible varieties and a discrete variety. Let $\varphi$ be a unital algebra isomorphism of $\mathcal{M}_{V}$ onto $\mathcal{M}_{W}$. Then there exist holomorphic maps $F$ and $G$ from $\mathbb{B}_{d}$ into $\mathbb{C}^{d}$ with coefficients in $\mathcal{M}_{d}$ such that

(1) $\left.F\right|_{W}=\left.\varphi^{*}\right|_{W}$ and $\left.G\right|_{V}=\left.\left(\varphi^{-1}\right)^{*}\right|_{V}$,

(2) $\left.G \circ F\right|_{W}=\operatorname{id}_{W}$ and $\left.F \circ G\right|_{V}=\operatorname{id}_{V}$,

(3) $\varphi(f)=f \circ F \quad$ for $f \in \mathcal{M}_{V}$, and

(4) $\varphi^{-1}(g)=g \circ G \quad$ for $g \in \mathcal{M}_{W}$.

Proof. First we show that $\varphi^{*}$ maps $W$ into $V$. Write

$$
W=D \cup W_{1} \cup \cdots \cup W_{n}
$$

where $D$ is discrete and each $W_{i}$ is an irreducible variety. The points in $D$ are isolated and thus are mapped into $V$ by Lemma 5.2. A minor modification of Corollary [5.4 (2) deals with the irreducible subvarieties. Since $D$ is a variety, there is a multiplier $k \in \mathcal{M}_{d}$ which vanishes on $D$ and is non-zero at a regular point $\lambda \in W_{1}$. Proceed as in the proof of the lemma, but define $f=h_{2} \ldots h_{n} k$. Then the argument is completed in the same manner. Reversing the roles of $V$ and $W$ shows that $\varphi^{*}$ maps $W$ onto $V$.

We have observed that $\varphi^{*}\left(\rho_{\lambda}\right)$ lies in the fiber over the point

$$
F(\lambda)=\left(\varphi\left(Z_{1}\right)(\lambda), \ldots, \varphi\left(Z_{d}\right)(\lambda)\right) .
$$

Since we now know that $\varphi^{*}$ maps $W$ into $V$, we see (with a slight abuse of notation) that $F=\left.\varphi^{*}\right|_{W}$. In particular, the coefficients of $F$ are multipliers. Thus by Proposition 2.6. each $f_{i}$ is the restriction to $W$ of a multiplier in $\mathcal{M}_{d}$, which we also denote by $f_{i}$. In particular, each $f_{i}$ is holomorphic on the entire ball $\mathbb{B}_{d}$. Thus (since $d<\infty$ ), $F$ is a bounded holomorphic function of the ball into $\mathbb{C}^{d}$. It may not carry $\mathbb{B}_{d}$ into itself, but we do have $F(W)=V$.

A similar argument applied to $\varphi^{-1}$ shows that $G(V) \subset W$ and $\left.G\right|_{V}=\left.\left(\varphi^{-1}\right)^{*}\right|_{V}$. Since $\left(\varphi^{-1}\right)^{*}=\left(\varphi^{*}\right)^{-1}$, we obtain that $\left.G \circ F\right|_{W}=\operatorname{id}_{W}$ and $\left.F \circ G\right|_{V}=\operatorname{id}_{V}$. The last two statements follow as in Lemma 4.2 .

Remark 5.7. Note that in the above theorem, the map $F$ can be chosen to be a polynomial if and only if the algebra homomorphism $\varphi$ takes the coordinate functions to (restrictions of) polynomials, and hence takes polynomials to polynomials. Likewise, $F$ can be chosen to have components which are continuous multipliers if and only if $\varphi$ takes the coordinate functions to continuous multipliers, and hence takes all continuous multipliers to continuous multipliers.

Remark 5.8. When $d=\infty$, there is no guarantee that the map $F$ constructed in our proof would actually have values in $\ell^{2}$. However, if we assume that $\varphi$ is completely 
bounded, then we can argue as follows. The row operator $Z=\left[\begin{array}{llll}Z_{1} & Z_{2} & Z_{3} & \ldots\end{array}\right]$ is a contraction. Thus $\varphi(Z)=\left[\varphi\left(Z_{1}\right) \varphi\left(Z_{2}\right) \varphi\left(Z_{3}\right) \ldots\right]$ is bounded by $\|\varphi\|_{c b}$. By Proposition 2.6. there are functions $f_{i} \in \mathcal{M}_{d^{\prime}}$ so that $\left.f_{i}\right|_{W}=\varphi\left(Z_{i}\right)$ and

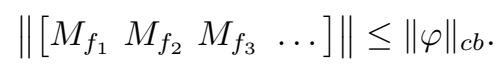

In particular, $F=\left[\begin{array}{llll}f_{1} & f_{2} & f_{3} & \ldots\end{array}\right]$ is bounded by $\|\varphi\|_{c b}$ in the sup norm. Theorem 5.6 can then be modified to apply in the case $d=\infty$. However, these hypotheses are very strong.

Corollary 5.9. Every algebraic automorphism of $\mathcal{M}_{d}$ for $d$ finite is completely isometric and is unitarily implemented.

Proof. The previous theorem shows that every automorphism is implemented as composition by a biholomorphic map of the ball onto itself, i.e. a conformal automorphism of $\mathbb{B}_{d}$. Proposition 4.1 shows that these automorphisms are completely isometric and unitarily implemented.

Now we consider the isometric case.

Theorem 5.10. Let $V$ and $W$ be varieties in $\mathbb{B}_{d}$, with $d<\infty$. Every isometric isomorphism of $\mathcal{M}_{V}$ onto $\mathcal{M}_{W}$ is completely isometric and thus is unitarily implemented.

Proof. Let $\varphi$ be an isometric isomorphism of $\mathcal{M}_{V}$ onto $\mathcal{M}_{W}$. By Corollary 5.4 (3), $\varphi^{*}$ maps $W$ onto $V$ and preserves the pseudohyperbolic distance. Let $F$ be the function constructed as in Theorem [5.6. As in Lemma 4.2 and Theorem [5.6, $F$ is a biholomorphism of $W$ onto $V$ and $\varphi(h)=h \circ F$.

After modifying both $V$ and $W$ by a conformal automorphism of the ball, we may assume that 0 belongs to both $V$ and $W$, and that $F(0)=0$. Set $w_{0}=0$ and choose a basis $w_{1}, \ldots, w_{k}$ for $\operatorname{span} W$. Let $v_{p}=F\left(w_{p}\right)$ for $1 \leq p \leq k$.

Suppose that $\left\|w_{p}\right\|=r_{p}$. This is the pseudohyperbolic distance to $w_{0}=0=$ $v_{0}$, so $\left\|v_{p}\right\|=r_{p}$ as well. Write $v_{p} / r_{p}=\sum_{j=1}^{d} c_{j} e_{j}$. Let $h_{p}(z)=\left\langle z, v_{p} / r_{p}\right\rangle=$ $\sum_{j=1}^{d} \bar{c}_{j} Z_{j}(z)$. This is a linear function on $V$, and thus lies in $\mathcal{M}_{V}$. Since $Z$ is a row contraction, $f$ has norm at most one. Therefore $k_{p}:=\varphi\left(h_{p}\right)=h_{p} \circ F$ has norm at most one in $\mathcal{M}_{W}$.

Now let $w_{k+1}=w$ be an arbitrary point in $W$, and set $v_{k+1}=v=F(w) \in V$. By a standard necessary condition for interpolation [2, Theorem 5.2], the fact that $\left\|k_{p}\right\| \leq 1$ means that in particular interpolating at the points $w_{0}, \ldots, w_{k}, w_{k+1}$, we obtain

$$
0 \leq\left[\frac{1-h_{p}\left(v_{i}\right) \overline{h_{p}\left(v_{j}\right)}}{1-\left\langle w_{i}, w_{j}\right\rangle}\right]_{0 \leq i, j \leq k+1} .
$$

In particular, look at the $3 \times 3$ minor using rows $0, p, k+1$ to obtain

$$
0 \leq\left[\begin{array}{ccc}
1 & 1 & 1 \\
1 & 1 & \frac{1-\overline{\left\langle v, v_{p}\right\rangle}}{1-\left\langle w_{p}, w\right\rangle} \\
1 & \frac{1-\left\langle v, v_{p}\right\rangle}{1-\left\langle w, w_{p}\right\rangle} & \frac{1-\left|\left\langle v, v_{p} / r_{p}\right\rangle\right|^{2}}{1-\|w\|^{2}}
\end{array}\right]
$$

By the Cholesky algorithm, we find that $\frac{1-\left\langle v, v_{p}\right\rangle}{1-\left\langle w, w_{p}\right\rangle}=1$. Therefore

$$
\left\langle v, v_{p}\right\rangle=\left\langle w, w_{p}\right\rangle \text { for } 1 \leq p \leq k .
$$


In particular, we obtain

$$
\left\langle v_{i}, v_{j}\right\rangle=\left\langle w_{i}, w_{j}\right\rangle \quad \text { for } 1 \leq i, j \leq k .
$$

Therefore there is a unitary operator $U$ acting on $\mathbb{C}^{d}$ such that $U w_{i}=v_{i}$ for $1 \leq i \leq k$. Now since $w \in W$ lies in $\operatorname{span}\left\{w_{1}, \ldots, w_{k}\right\}$, it is uniquely determined by the inner products $\left\langle w, w_{i}\right\rangle$ for $1 \leq i \leq k$. Since $v$ has the same inner products with $v_{1}, \ldots, v_{k}$, we find that $U w=P_{N} v$, where $N=\operatorname{span}\left\{v_{1}, \ldots, v_{k}\right\}$. However, we also have

whence $v=U w$.

$$
\|v\|=\|w\|=\|U w\|=\left\|P_{N} v\right\|
$$

Therefore $F$ agrees with the unitary $U$, and hence $\varphi$ is implemented by an automorphism of the ball. So by Proposition 4.1 $\varphi$ is completely isometric and is unitarily implemented.

With these results in hand, we may repeat the arguments in [16, Section 11.3] word for word to obtain the following automatic continuity result. Recall that the weak-operator and the weak-* topologies on $\mathcal{M}_{V}$ coincide by Lemma 3.1

Theorem 5.11. Let $\varphi: \mathcal{M}_{V} \rightarrow \mathcal{M}_{W}$, for $d<\infty$, be a unital algebra isomorphism given by composition: $\varphi(h)=h \circ F$, where $F$ is a holomorphic map of $W$ onto $V$ whose coefficients are multipliers. Then $\varphi$ is continuous with respect to the weakoperator and the weak-* topologies.

\section{EXAMPLES}

In this section, we examine a possible converse to Theorem 5.6 in the context of a number of examples. What we find is that the desired converse is not always true. That is, suppose that $V$ and $W$ are varieties in $\mathbb{B}_{d}$, and $F$ and $G$ are holomorphic functions on the ball satisfying the conclusions of Theorem 5.6. We are interested in when this implies that the algebras $\mathcal{M}_{V}$ and $\mathcal{M}_{W}$ are isomorphic.

Finitely many points in the ball. Let $V=\left\{v_{1}, \ldots, v_{n}\right\} \subseteq \mathbb{B}_{d}$. Then $\mathcal{A}_{V}=\mathcal{M}_{V}$ and they are both isomorphic to $\ell_{n}^{\infty}=C(V)$. The characters are evaluations at points of $V$. If $W$ is another $n$ point set in $\mathbb{B}_{d^{\prime}}$, then $\mathcal{M}_{W}$ is isomorphic to $\mathcal{M}_{V}$. Also, there are (polynomial) maps $f: \mathbb{B}_{d} \rightarrow \mathbb{C}^{d^{\prime}}$ and $g: \mathbb{B}_{d^{\prime}} \rightarrow \mathbb{C}^{d}$ which are inverses of one another when restricted to $V$ and $W$. Additionally, if $W$ is an $m$ point set, $m \neq n$, then obviously $\mathcal{M}_{V}$ is not isomorphic to $\mathcal{M}_{W}$, and there also exists no biholomorphism. In this simple case we see that $\mathcal{M}_{V} \cong \mathcal{M}_{W}$ if and only if there exists a biholomorphism, and this happens if and only if $|W|=|V|$.

Nevertheless, the situation for finite sets is not ideal. Let $V$ and $W$ be finite subsets of the ball, and let $F: W \rightarrow V$ be a biholomorphism. It is natural to hope that the norm of the induced isomorphism can be bounded in terms of the multiplier norm of $F$. The following example shows that this is not possible.

Example 6.1. Fix $n \in \mathbb{N}$ and $r \in(0,1)$. Put $\xi=\exp \left(\frac{2 \pi i}{n}\right)$ and let

$$
V=\{0\} \cup\left\{r \xi^{j}\right\}_{j=1}^{n}
$$

and

$$
W=\{0\} \cup\left\{\frac{r}{2} \xi^{j}\right\}_{j=1}^{n} .
$$


The map $F(z)=2 z$ is a biholomorphism of $W$ onto $V$ that extends to an $H^{\infty}$ function of multiplier norm 2. We will show that the norm of the induced isomorphism $\mathcal{M}_{V} \rightarrow \mathcal{M}_{W}$, given by $f \mapsto f \circ F$, is at least $2^{n}$.

Consider the following function in $\mathcal{M}_{V}$ :

$$
f(0)=0 \quad \text { and } \quad f\left(r \xi^{j}\right)=r^{n} \quad \text { for } 1 \leq j \leq n .
$$

We claim that the multiplier norm of $f$ is 1 . By Proposition 2.6, $\|f\|$ is the minimal norm of an $H^{\infty}$ function that interpolates $f$. The function $g(z)=z^{n}$ certainly interpolates and has norm 1 . We will show that it is of minimal norm.

The Pick matrix associated to the problem of interpolating $f$ on $V$ by an $H^{\infty}$ function of norm 1 is

$$
\left[\begin{array}{ccccc}
1 & 1 & 1 & \cdots & 1 \\
1 & \frac{1-r^{2 n}}{1-r^{2} \xi \bar{\xi}} & \frac{1-r^{2 n}}{1-r^{2} \xi \overline{\xi^{2}}} & \cdots & \frac{1-r^{2 n}}{1-r^{2} \xi \overline{\xi^{n}}} \\
1 & \frac{1-r^{2 n}}{1-r^{2} \xi^{2} \bar{\xi}} & \frac{1-r^{2 n}}{1-r^{2} \xi^{2} \overline{\xi^{2}}} & \cdots & \frac{1-r^{2 n}}{1-r^{2} \xi^{2} \overline{\xi^{n}}} \\
\vdots & \vdots & \vdots & \ddots & \vdots \\
1 & \frac{1-r^{2 n}}{1-r^{2} \xi^{n} \bar{\xi}} & \frac{1-r^{2 n}}{1-r^{2} \xi^{n} \overline{\xi^{2}}} & \cdots & \frac{1-r^{2 n}}{1-r^{2} \xi^{n} \overline{\xi^{n}}}
\end{array}\right] .
$$

To show that $g$ is the (unique) function of minimal norm that interpolates $f$, it suffices to show that this matrix is singular. (We are using well-known facts about Pick interpolation. See Chapter 6 in 2.)

We will show that the lower right principal submatrix

$$
A=\left[\frac{1-r^{2 n}}{1-r^{2} \xi^{i} \overline{\xi^{j}}}\right]_{i, j=1}^{n}
$$

has the vector $(1, \ldots, 1)^{t}$ as an eigenvector with eigenvalue $n$. It follows that $(n,-1,-1, \ldots,-1)^{t}$ is in the kernel of the Pick matrix. (The matrix $A$ is invertible, so the Pick matrix has rank $n$.)

Indeed, for any $i$,

$$
\begin{aligned}
\sum_{j=1}^{n} \frac{1-r^{2 n}}{1-r^{2} \xi^{i} \overline{\xi^{j}}} & =\left(1-r^{2 n}\right) \sum_{j=1}^{n} \sum_{k=0}^{\infty}\left(r^{2} \xi^{i} \overline{\xi^{j}}\right)^{k} \\
& =\left(1-r^{2 n}\right) \sum_{k=0}^{\infty} \sum_{j=1}^{n} r^{2 k} \xi^{i k} \overline{\xi^{j k}} \\
& =\left(1-r^{2 n}\right) \sum_{m=0}^{\infty} n r^{2 m n} \xi^{i m n} \\
& =n \frac{1-r^{2 n}}{1-r^{2 n}}=n .
\end{aligned}
$$

We used the familiar fact that $\sum_{j=1}^{n} \xi^{j k}$ is equal to $n$ for $k \equiv 0(\bmod n)$ and equal to 0 otherwise. Therefore $\|f\|=1$.

Now we will show that $f \circ F \in \mathcal{M}_{W}$ has norm $2^{n}$, where $F(z)=2 z$. The function $f \circ F$ is given by

$$
f \circ F(0)=0 \quad \text { and } \quad f \circ F\left(\frac{r}{2} \xi^{j}\right)=r^{n} \quad \text { for } 1 \leq j \leq n .
$$


The unique $H^{\infty}$ function of minimal norm that interpolates $f \circ F$ is $h(z)=2^{n} z^{n}$. This follows from precisely the same reasoning as above. Therefore the isomorphism has norm at least $2^{n}$.

Blaschke sequences. We will now provide an example of two discrete varieties which are biholomorphic but yield non-isomorphic algebras.

Example 6.2. Let

$$
v_{n}=1-1 / n^{2} \quad \text { and } \quad w_{n}=1-e^{-n^{2}} \quad \text { for } n \geq 1 .
$$

Set $V=\left\{v_{n}\right\}_{n=1}^{\infty}$ and $W=\left\{w_{n}\right\}_{n=1}^{\infty}$. Both $V$ and $W$ satisfy the Blaschke condition, so they are analytic varieties in $\mathbb{D}$. Let $B(z)$ be the Blaschke product with simple zeros at points in $W$. Define

$$
h(z)=1-e^{\frac{1}{z-1}}
$$

and

Then $g, h \in H^{\infty}$ and they satisfy

$$
g(z)=\frac{\log (1-z)+1}{\log (1-z)}\left(1-\frac{B(z)}{B(0)}\right) .
$$

$$
\left.h \circ g\right|_{W}=\operatorname{id}_{W} \quad \text { and }\left.g \circ h\right|_{V}=\operatorname{id}_{V} .
$$

However, by the corollary in [22, p. 204], $W$ is an interpolating sequence and $V$ is not. Thus the algebras $\mathcal{M}_{V}$ and $\mathcal{M}_{W}$ cannot be similar by a map sending normalized kernel functions to normalized kernel functions. The reason is that the normalized kernel functions corresponding to an interpolating sequence form a Riesz system, while those corresponding to a non-interpolating sequence do not. In fact, $\mathcal{M}_{V}$ and $\mathcal{M}_{W}$ cannot be isomorphic via any isomorphism, as we see below.

Theorem 6.3. Let $V=\left\{v_{n}\right\}_{n=1}^{\infty} \subseteq \mathbb{B}_{d}$, with $d<\infty$, be a sequence satisfying the Blaschke condition $\sum\left(1-\left\|v_{n}\right\|\right)<\infty$. Then $\mathcal{M}_{V}$ is isomorphic to $\ell^{\infty}$ if and only if $V$ is interpolating.

Proof. By definition, $V$ is interpolating if and only if $\mathcal{M}_{V}$ is isomorphic to $\ell^{\infty}$ via the restriction map. It remains to prove that if $V$ is not an interpolating sequence, then $\mathcal{M}_{V}$ cannot be isomorphic to $\ell^{\infty}$ via any other isomorphism.

Let $V$ be a non-interpolating sequence, and let $W$ be any interpolating sequence. If $\mathcal{M}_{V}$ is isomorphic to $\ell^{\infty}$, then it is isomorphic to $\mathcal{M}_{W}$. But by Lemma 5.2. this isomorphism must be implemented by composition with a holomorphic map, showing that $\mathcal{M}_{V}$ is isomorphic to $\ell^{\infty}$ via the restriction map. This is a contradiction.

Remark 6.4. We require the Blaschke condition to insure that $V$ is a variety of the type we consider, i.e., a zero set of an ideal of multipliers (see [5, Theorem 1.11]). Any discrete variety in $\mathbb{D}$ satisfies this condition.

Curves. Let $V$ be a variety in $\mathbb{B}_{d}$. If $\mathcal{M}_{V}$ is isomorphic to $H^{\infty}(\mathbb{D})$, then by Theorem 5.6 we know that $V$ must be biholomorphic to the disc. To study the converse implication, we shall start with a disc biholomorphically embedded in a ball and try to establish a relationship between the associated algebras $\mathcal{M}_{V}$ and its reproducing kernel Hilbert space $\mathcal{F}_{V}$ and $H^{\infty}(\mathbb{D})$ and $H^{2}(\mathbb{D})$.

Suppose that $h$ is a holomorphic map from the disc $\mathbb{D}$ into $\mathbb{B}_{d}$ such that $h(\mathbb{D})=V$, and that there exists a holomorphic map $g: \mathbb{B}_{d} \rightarrow \mathbb{C}$ such that $\left.g\right|_{V}=h^{-1}$. 
The following result shows that in many cases, the desired isomorphism exists [3]. See [4, §2.3.6] for a strengthening to planar domains and for a technical correction.

Theorem 6.5 (Alpay-Putinar-Vinnikov). Suppose that $h$ is an injective holomorphic function of $\mathbb{D}$ onto $V \subset \mathbb{B}_{d}$ such that

(1) $h$ extends to a $C^{1}$ function on $\overline{\mathbb{D}}$,

(2) $\|h(z)\|=1$ if and only if $|z|=1$,

(3) $\left\langle h(z), h^{\prime}(z)\right\rangle \neq 0$ when $|z|=1$.

Then $\mathcal{M}_{V}$ is isomorphic to $H^{\infty}$.

Condition (3) should be seen as saying that $V$ meets the boundary of the ball non-tangentially. We do not know whether such a condition is necessary.

The authors of 3 were concerned with extending multipliers on $V$ to multipliers on the ball. This extension follows from Proposition 2.6.

By the results of Section 4, there is no loss of generality in assuming that $h(0)=$ 0 , and we do so. Define a kernel $\tilde{k}$ on $\mathbb{D}$ by

$$
\tilde{k}(z, w)=k(h(z), h(w))=\frac{1}{1-\langle h(z), h(w)\rangle} .
$$

Let $\mathcal{H}$ be the RKHS determined by $\tilde{k}$. Write $\tilde{k}_{w}$ for the function $\tilde{k}(\cdot, w)$.

The following routine lemma shows that we can consider this new kernel on the disc instead of $\mathcal{F}_{V}$.

Lemma 6.6. The map $\tilde{k}_{z} \mapsto k_{h(z)}$ extends to a unitary map $U$ of $\mathcal{H}$ onto $\mathcal{F}_{V}$. Hence, the multiplier algebra $\operatorname{Mult}(\mathcal{H})$ is unitarily equivalent to $\mathcal{M}_{V}$. This equivalence is implemented by composition with $h$ :

$$
U^{*} M_{f} U=M_{f \circ h} \quad \text { for } f \in \mathcal{M}_{V} .
$$

Proof. A simple computation shows that

$$
\left\|\sum_{i} c_{i} \tilde{k}_{z_{i}}\right\|^{2}=\sum_{i, j} \frac{c_{i} \overline{c_{j}}}{1-\left\langle h\left(z_{i}\right), h\left(z_{j}\right)\right\rangle}=\left\|\sum_{i} c_{i} k_{h\left(z_{i}\right)}\right\|^{2} .
$$

So we get a unitary $U: \mathcal{H} \rightarrow \mathcal{F}_{V}$. As in the proof of Proposition 4.1, for all $f \in \mathcal{M}_{V}$ we have $U^{*} M_{f} U=M_{f \circ h}$.

Our goal in this section is to study conditions on $h$ which yield a natural isomorphism of the RKHSs $\mathcal{H}$ and $H^{2}(\mathbb{D})$. The first result is that the Szego kernel $k_{z}$ dominates the kernel $\tilde{k}_{z}$.

Lemma 6.7. Suppose that $h$ is a holomorphic map of $\mathbb{D}$ into $\mathbb{B}_{d}$. Then for any finite subset $\left\{z_{1}, \ldots, z_{n}\right\} \subset \mathbb{D}$,

$$
\left[\frac{1}{1-\left\langle h\left(z_{j}\right), h\left(z_{i}\right)\right\rangle}\right] \leq\left[\frac{1}{1-z_{j} \overline{z_{i}}}\right] .
$$

Proof. Observe that $h(z) / z$ maps $\mathbb{D}$ into $\overline{\mathbb{B}_{d}}$ by Schwarz's Lemma [27, Theorem 8.1.2]. Thus by the matrix version of the Nevanlinna-Pick Theorem for the unit disk, we obtain that

$$
0 \leq\left[\frac{1-\left\langle h\left(z_{j}\right) / z_{j}, h\left(z_{i}\right) / z_{i}\right\rangle}{1-z_{j} \overline{z_{i}}}\right]=\left[\frac{1}{z_{j} \overline{z_{i}}}\right] \circ\left[\frac{1-\left\langle h\left(z_{j}\right), h\left(z_{i}\right)\right\rangle}{1-z_{j} \overline{z_{i}}}-1\right] .
$$


Here $\circ$ represents the Schur product. But $\left[\frac{1}{z_{j} \overline{z_{i}}}\right]$ and its Schur inverse $\left[z_{j} \overline{z_{i}}\right]$ are positive. Therefore the second matrix on the right is positive. This can be rewritten as

$$
[1] \leq\left[\frac{1-\left\langle h\left(z_{j}\right), h\left(z_{i}\right)\right\rangle}{1-z_{j} \overline{z_{i}}}\right]
$$

where [1] represents an $n \times n$ matrix of all 1's. Now

$$
\left[\frac{1}{1-\left\langle h\left(z_{j}\right), h\left(z_{i}\right)\right\rangle}\right]=\left[\left\langle\tilde{k}_{z_{i}}, \tilde{k}_{z_{j}}\right\rangle\right] \geq 0 .
$$

So the Schur multiplication by this operator to the previous inequality yields

$$
\left[\frac{1}{1-\left\langle h\left(z_{j}\right), h\left(z_{i}\right)\right\rangle}\right] \leq\left[\frac{1}{1-z_{j} \overline{z_{i}}}\right] .
$$

We obtain the well-known consequence that there is a contractive map of $H^{2}$ into $\mathcal{H}$.

Proposition 6.8. The linear map $R$, defined by $R k_{z}=\tilde{k}_{z}$ for $z \in \mathbb{D}$, from $\operatorname{span}\left\{k_{z}: z \in \mathbb{D}\right\}$ to $\operatorname{span}\left\{\tilde{k}_{z}: z \in \mathbb{D}\right\}$ extends to a contractive map from $H^{2}$ into $\mathcal{H}$.

Proof. This follows from an application of Lemma 6.7. Given $a_{i} \in \mathbb{C}$, let $\mathbf{a}=$ $\left(a_{1}, \ldots, a_{n}\right)^{t}$. Observe that

$$
\begin{aligned}
\left\|R \sum_{i=1}^{n} a_{i} k_{z_{i}}\right\|^{2} & =\left\|\sum_{i=1}^{n} a_{i} \tilde{k}_{z_{i}}\right\|^{2}=\sum_{i, j=1}^{n} a_{i} \overline{a_{j}}\left\langle\tilde{k}_{z_{i}}, \tilde{k}_{z_{j}}\right\rangle \\
& =\left\langle\left[\left\langle\tilde{k}_{z_{i}}, \tilde{k}_{z_{j}}\right\rangle\right] \mathbf{a}, \mathbf{a}\right\rangle \leq\left\langle\left[\left\langle k_{z_{i}}, k_{z_{j}}\right\rangle\right] \mathbf{a}, \mathbf{a}\right\rangle \\
& =\sum_{i, j=1}^{n} a_{i} \overline{a_{j}}\left\langle k_{z_{i}}, k_{z_{j}}\right\rangle=\left\|\sum_{i=1}^{n} a_{i} k_{z_{i}}\right\|^{2} .
\end{aligned}
$$

Hence $R$ is contractive and extends to $H^{2}$ by continuity.

Example 6.9. Let $h: \mathbb{D} \rightarrow \mathbb{B}_{d}$ be given by

$$
h(z)=\left(a_{1} z, a_{2} z^{n_{2}}, \ldots, a_{d} z^{n_{d}}\right),
$$

where $a_{1} \neq 0$ and $\sum_{l=1}^{d}\left|a_{l}\right|^{2}=1$. Let $V=h(\mathbb{D})$. Then $\mathcal{M}_{V}$ is similar to $H^{\infty}(\mathbb{D})$, and $\mathcal{M}_{V}=H^{\infty}(V)$. Moreover, $\mathcal{A}_{V}$ is similar to $\mathrm{A}(\mathbb{D})$. This follows from Theorem 6.5. but we will provide a direct argument.

First observe that for $p \geq N=\max \left\{n_{l}: 1 \leq l \leq d\right\}$, we have

$$
\begin{aligned}
\frac{\langle h(z), h(w)\rangle-z^{p} \bar{w}^{p}}{1-z \bar{w}} & =\sum_{l=1}^{d}\left|a_{l}\right|^{2}\left(\frac{z^{n_{l}} \bar{w}^{n_{l}}-z^{p} \bar{w}^{p}}{1-z \bar{w}}\right) \\
& =\sum_{l=1}^{d}\left|a_{l}\right|^{2} z^{n_{l}} \bar{w}^{n_{l}}\left(\frac{1-z^{p-n_{l}} \bar{w}^{p-n_{l}}}{1-z \bar{w}}\right) .
\end{aligned}
$$

Therefore if $z_{1}, \ldots, z_{k}$ are distinct points in $\mathbb{D}$, the $k \times k$ matrix

$$
A_{p}:=\left[\frac{\left\langle h\left(z_{i}\right), h\left(z_{j}\right)\right\rangle-z_{i}^{p} \bar{z}_{j}^{p}}{1-z_{i} \bar{z}_{j}}\right]=\sum_{l=1}^{d}\left|a_{l}\right|^{2}\left[z_{i}^{n_{l}} \bar{z}_{j}^{n_{l}}\right] \circ\left[\frac{1-z_{i}^{p-n_{l}} \bar{z}_{j}^{p-n_{l}}}{1-z \bar{w}}\right]
$$


is positive definite because the second matrix on the right is positive by Pick's condition, and the Schur product of positive matrices is positive.

Since the first coordinate of $h$ is injective, we see that $h$ is injective. Moreover,

$$
\left\|h^{-1}\right\|_{\mathcal{M}_{V}} \leq\left\|a_{1}^{-1} z_{1}\right\|_{\mathcal{M}}=\left|a_{1}\right|^{-1}=: C .
$$

Since the kernel for $\mathcal{F}_{V}$ is a complete NP kernel, applying this to $\left(h^{-1}\right)^{2^{n-1}}$ yields the positivity of the matrices

$$
\left[\frac{C^{2^{n}}-z_{i}^{2^{n-1}} \bar{z}_{j}^{2^{n-1}}}{1-\left\langle h\left(z_{i}\right), h\left(z_{j}\right)\right\rangle}\right]
$$

Since $z^{2^{n-1}}$ has norm one, the Pick condition shows that

$$
\left[\frac{1-z_{i}^{2^{n-1}} \bar{z}_{j}^{2^{n-1}}}{1-z_{i} \bar{z}_{j}}\right] \geq 0
$$

Thus we obtain positive matrices

$$
\begin{aligned}
H_{n} & :=\left[\frac{C^{2^{n}}-z_{i}^{2^{n-1}} \bar{z}_{j}^{2^{n-1}}}{1-\left\langle h\left(z_{i}\right), h\left(z_{j}\right)\right\rangle}\right] \circ\left[\frac{1-z_{i}^{2^{n-1}} \bar{z}_{j}^{2^{n-1}}}{1-z_{i} \bar{z}_{j}}\right] \\
& =\left[\frac{C^{2^{n}}-\left(C^{2^{n}}+1\right) z_{i}^{2^{n-1}} \bar{z}_{j}^{2^{n-1}}+z_{i}^{2^{n}} \bar{z}_{j}^{2^{n}}}{\left(1-\left\langle h\left(z_{i}\right), h\left(z_{j}\right)\right\rangle\right)\left(1-z_{i} \bar{z}_{j}\right)}\right] .
\end{aligned}
$$

Choose $M$ so that $2^{M} \geq N$. We form a telescoping sum of positive multiples of the $H_{n}$ 's:

$$
0 \leq \sum_{n=1}^{M} b_{n} H_{n}=\left[\frac{(D-1)-D z_{i} \bar{z}_{j}+z_{i}^{2^{M}} \bar{z}_{j}^{2^{M}}}{\left(1-\left\langle h\left(z_{i}\right), h\left(z_{j}\right)\right\rangle\right)\left(1-z_{i} \bar{z}_{j}\right)}\right]=: H
$$

where $b_{M}=1, b_{n}=\prod_{k=n+1}^{M}\left(C^{2^{k}}+1\right)$ for $1 \leq n<M$ and $D=\prod_{k=1}^{M}\left(C^{2^{k}}+1\right)$. Thus

$$
\begin{aligned}
{\left[\frac{D}{1-\left\langle h\left(z_{i}\right), h\left(z_{j}\right)\right\rangle}\right]-\left[\frac{1}{1-z_{i} \bar{z}_{j}}\right] } & =\left[\frac{(D-1)-D z_{i} \bar{z}_{j}+\left\langle h\left(z_{i}\right), h\left(z_{j}\right)\right\rangle}{\left(1-\left\langle h\left(z_{i}\right), h\left(z_{j}\right)\right\rangle\right)\left(1-z_{i} \bar{z}_{j}\right)}\right] \\
& =H+A_{2^{M}} \circ\left[\frac{1}{1-\left\langle h\left(z_{i}\right), h\left(z_{j}\right)\right\rangle}\right] \geq 0 .
\end{aligned}
$$

This inequality shows that the two kernels $k_{z}$ and $\tilde{k}_{z}$ are comparable. The argument of Proposition 6.8 shows that $\left\|R^{-1}\right\| \leq D$. In particular, $R$ yields an isomorphism of the two RKHSs $H^{2}$ and $\mathcal{H}$. This yields the desired isomorphism of $H^{\infty}$ and $\mathcal{M}_{V}$.

This isomorphism is not isometric. Indeed, if it were, then we would have $\left\|h^{-1}\right\|_{\mathcal{M}_{V}}=\|z\|_{\infty}=1$. This would imply that

$$
0 \leq\left[\frac{1-z_{i} \bar{z}_{j}}{1-\left\langle h\left(z_{i}\right), h\left(z_{j}\right)\right\rangle}\right] .
$$

Thus arguing as in Lemma 6.7, we obtain

$$
\left[\frac{1}{1-z_{j} \overline{z_{i}}}\right] \leq\left[\frac{1}{1-\left\langle h\left(z_{j}\right), h\left(z_{i}\right)\right\rangle}\right] .
$$

But then the map $R$ would be unitary and the algebras would be completely isometric. So by Lemma 4.3 the map $h$ would map onto an affine disk-which it does not. 
A class of examples in $\mathbb{B}_{\infty}$. We will now exhibit biholomorphisms of $\mathbb{D}$ into $\mathbb{B}_{\infty}$, some of which yield an isomorphism and some which do not.

Let $\left\{b_{n}\right\}_{n=1}^{\infty}$ be a sequence of complex numbers with $\sum\left|b_{n}\right|^{2}=1$ and $b_{1} \neq 0$. Let $h: \mathbb{D} \rightarrow \mathbb{B}_{\infty}$ be given by

$$
h(z)=\left(b_{1} z, b_{2} z^{2}, b_{3} z^{3}, \ldots\right) .
$$

Note that $h$ is analytic (because it is given by a power series in the disc), with the analytic inverse

$$
g\left(z_{1}, z_{2}, z_{3}, \ldots\right)=z_{1} / b_{1} .
$$

The set $V=h(\mathbb{D})$ is the variety in $\mathbb{B}_{\infty}$ determined by the equations

$$
z_{k}=\frac{b_{k}}{b_{1}} z_{1}^{k} \quad \text { for } k \geq 2 \text {. }
$$

As above let

$$
\tilde{k}(z, w)=\frac{1}{1-\langle h(z), h(w)\rangle},
$$

and let $\mathcal{H}$ be the RKHS determined by $\tilde{k}$. By Lemma 6.6, $\mathcal{H}$ is equivalent to $\mathcal{F}_{V}$. The special form of $h$ allows us to write

$$
\frac{1}{1-\langle h(z), h(w)\rangle}=\sum_{n=0}^{\infty}\left(\sum_{i=1}^{\infty}\left|b_{i}\right|^{2} z^{i} \bar{w}^{i}\right)^{n}=\sum_{n=0}^{\infty} a_{n}(z \bar{w})^{n} .
$$

By a basic result in RKHSs, $\tilde{k}(z, w)=\sum e_{n}(z) \overline{e_{n}(w)}$, where $\left\{e_{n}\right\}$ is an orthonormal basis for $\tilde{k}$ (see Proposition 2.18 of [2]). Hence $\mathcal{H}$ is the space of holomorphic functions on $\mathbb{D}$ with orthonormal basis $\left\{\sqrt{a_{n}} z^{n}\right\}_{n=0}^{\infty}$.

The map $R$ defined in Proposition 6.8 is a contraction. Observe that $R^{*}: \mathcal{H} \rightarrow$ $H^{2}$ is given by composition with the identity mapping because

$$
\left(R^{*} f\right)(z)=\left\langle R^{*} f, k_{z}\right\rangle=\left\langle f, R k_{z}\right\rangle=\left\langle f, \tilde{k}_{z}\right\rangle=f(z) .
$$

It is easy to see that the issue is whether $R$ is bounded below. Since $\left\|z^{n}\right\|_{H^{2}}=1$ and $\left\|z^{n}\right\|_{\mathcal{H}}=1 / \sqrt{a_{n}}$, we get:

Proposition 6.10. $\mathcal{H}$ is equivalent to $H^{2}$ via $R$ if and only if there are constants $0<c<C$ so that $c \leq a_{n} \leq C$ for $n \geq 0$.

The coefficients $a_{n}$ are determined by the sequence $\left\{\left|b_{n}\right|\right\}_{n=1}^{\infty}$ and can be found recursively by the formulae

$$
a_{0}=1 \quad \text { and } \quad a_{n}=\left|b_{1}\right|^{2} a_{n-1}+\ldots+\left|b_{n}\right|^{2} a_{0} \text { for } n \geq 1 .
$$

The logic behind this recursion is that the term $a_{n}(z \bar{w})^{n}$ gets contributions from the sum

$$
\sum_{k=1}^{n}\left(\sum_{i=1}^{n}\left|b_{i}\right|^{2} z^{i} \bar{w}^{i}\right)^{k}=\left(\sum_{i=1}^{n}\left|b_{i}\right|^{2} z^{i} \bar{w}^{i}\right) \sum_{k=1}^{n}\left(\sum_{i=1}^{n}\left|b_{i}\right|^{2} z^{i} \bar{w}^{i}\right)^{k-1} .
$$

Every $\left|b_{i}\right|^{2} z^{i} \bar{w}^{i}$ from the factor $\sum_{i=1}^{n}\left|b_{i}\right|^{2} z^{i} \bar{w}^{i}$ needs to be matched with the $(z \bar{w})^{n-i}$ term from the factor $\sum_{k=1}^{n}\left(\sum_{i=1}^{n}\left|b_{i}\right|^{2} z^{i} \bar{w}^{i}\right)^{k-1}$, which has coefficient precisely $a_{n-i}$. It follows by induction from equation (6.1) that $a_{n} \leq 1$. This provides an alternative proof of Proposition 6.8 in this special case.

We will now construct a sequence $\left\{b_{n}\right\}_{n=1}^{\infty}$ that makes lim inf $a_{n}>0$, and another sequence that makes $\lim \inf a_{n}=0$. By Proposition 6.10, this will show that there 
are choices of $\left\{b_{n}\right\}_{n=1}^{\infty}$ for which $\mathcal{H}$ and $H^{2}$ are naturally isomorphic, and there are choices for which they are not.

Example 6.11. Define $b_{n}=(1 / 2)^{n / 2}$ for $n \geq 1$. It follows from the recursion relation (6.1) that $a_{n}=1 / 2$ for $n>1$. Thus $R^{*}$ is bounded below, showing that $\mathcal{H}$ and $H^{2}$ are naturally isomorphic.

Example 6.12. We will choose a rapidly increasing sequence $\left\{n_{k}\right\}_{k=1}^{\infty}$ with $n_{1}=1$ and define the sequence $\left\{b_{n}\right\}_{n=1}^{\infty}$ by

$$
b_{m}= \begin{cases}(1 / 2)^{k / 2} & \text { if } m=n_{k}, \\ 0 & \text { otherwise } .\end{cases}
$$

The sequence $\left\{n_{k}\right\}_{k=1}^{\infty}$ will be defined recursively so that $a_{n_{k}-1} \leq 1 / k$.

We begin with $n_{1}=1$ and $a_{0}=1$. Suppose that we have already chosen $n_{1}, \ldots, n_{k}$. This means that we have already determined the sequence $b_{1}, \ldots, b_{n_{k}}$, but the tail $b_{n_{k}+1}, b_{n_{k}+2}, \ldots$ is yet to be determined. We compute

$$
\sum_{m=1}^{n_{k}} b_{m}^{2}=\sum_{j=1}^{k} b_{n_{j}}^{2}=\sum_{j=1}^{k} 1 / 2^{j}=r<1 .
$$

Thus, if $b_{n_{k}+1}=b_{n_{k}+2}=\ldots=b_{(N+1) n_{k}}=0$, then it follows from (6.1) that $a_{(N+1) n_{k}} \leq r^{N}$ (recall that $a_{n} \leq 1$ for all $\left.n\right)$. Therefore we may choose $N$ so large that $a_{(N+1) n_{k}} \leq(k+1)^{-1}$, and we set $n_{k+1}=(N+1) n_{k}+1$.

Our construction yields a sequence $\left\{b_{n}\right\}_{n=1}^{\infty}$ so that $\liminf a_{n}=0$. Thus the kernel for the analytic disk $V$ so defined is not similar to $H^{2}$.

We do not know whether $\mathcal{M}_{V}$ is isomorphic to $H^{\infty}$ or not. We suspect that it isn't.

Remark 6.13. Suppose that there is some $N$ such that $b_{n}=0$ for all $n>N$. Then the mapping $h: \mathbb{D} \rightarrow \mathbb{B}_{\infty}$ given by

$$
h(z)=\left(b_{1} z, b_{2} z^{2}, b_{3} z^{3}, \ldots\right)
$$

can be considered as a mapping into $\mathbb{B}_{N}$. Equation (6.1) implies that for $n>N$, $a_{n}$ will always remain between the minimum and the maximum of $a_{0}, a_{1}, \ldots, a_{N}$. Therefore, the conditions of Proposition 6.10 are fulfilled, and $\mathcal{H}$ is equivalent to $H^{2}$ via $R$. This is an alternate argument to obtain Example 6.9

\section{The NORM Closed algebras $\mathcal{A}_{V}$}

Let $\mathcal{A}_{V}$ be the norm closure of the polynomials in $\mathcal{M}_{V}$. Define a closed ideal $I_{V}$ of $\mathcal{A}_{V}$ by

$$
I_{V}=\{f \in \mathcal{A}: f(\lambda)=0 \text { for all } \lambda \in V\} .
$$

It is natural to study the algebra $\mathcal{A}_{V}$ and the quotient algebra $\mathcal{A} / I_{V}$, and to ask whether these algebras can be identified. We make a few remarks about the subtleties involved. These subtleties are the reason why in general it seems that the algebras $\mathcal{M}_{V}$ will be more amenable to general study.

Consider the following special assumption, which we will usually assume when considering $\mathcal{A}_{V}$ :

$$
\left[I_{V} H_{d}^{2}\right]=\left[J_{V} H_{d}^{2}\right]
$$

By Theorem 2.4, this is equivalent to assuming that the woT-closure of $I_{V}$ is $J_{V}$. In function theoretic terms, this means that every $f \in J_{V}$ is the bounded pointwise 
limit of a net of functions in $I_{V}$. It is not clear when this happens in general. A large class of varieties for which this condition holds is the class of homogeneous varieties [16, Section 6]. Another class is described below.

In dimension $d=1$, the analytic varieties are sequences of points which are either finite or satisfy the Blaschke condition. For such a sequence $V$, let us denote $S(V)=\bar{V} \cap \mathbb{T}$. When the Lebesgue measure of $S(V)$ is positive, there is no non-zero $f \in A(\mathbb{D})$ that vanishes on $V$ because a non-zero function in the disk algebra must be non-zero a.e. on the unit circle. So $I_{V}=0$. On the other hand, $J_{V} \neq 0$, because it contains the Blaschke product of the sequence $V$. So it cannot be the WOTclosure of $I_{V}$. In particular, the special assumption (7.1) is not always satisfied. If the Lebesgue measure $|S(V)|$ of $S(V)$ is zero, then the special assumption is valid.

Lemma 7.1. Let $V$ be an analytic variety in $\mathbb{D}$ such that $S(V)$ has zero measure. Then the ideal $J_{V}$ is the wOT-closure of $I_{V}$.

Proof. Let $B$ be the Blaschke product with simple zeros on $V$. It suffices to construct, for every $f \in J_{V}=B H^{\infty}$, a bounded sequence in $I_{V}$ converging pointwise to $f$. Factor $f=B h$ with $h \in H^{\infty}$. By a theorem of Fatou there is an analytic function $g$ with $\operatorname{Re} g \geq 0$ such that $e^{-g}$ is in $A(\mathbb{D})$ and vanishes precisely on $S(V)$. Define

$$
f_{n}(z)=B(z) e^{-g(z) / n} h\left(\left(1-\frac{1}{n}\right) z\right) \quad \text { for } n \geq 1 .
$$

This sequence belongs to $\mathrm{A}(\mathbb{D})$, is bounded by $\|f\|_{\infty}$, and converges to $f$ uniformly on compact subsets of the disk. Hence it converges to $f$ in the WOT topology.

The importance of the special assumption (17.1) is in the following result.

Proposition 7.2. Let $V$ be an ideal such that $\left[I_{V} H_{d}^{2}\right]=\left[J_{V} H_{d}^{2}\right]$. Let $\mathcal{A}_{V}$ be the norm closure of the polynomials in $\mathcal{M}_{V}$. Then

(1) For every $f \in \mathcal{A}_{d}$, the compression of $M_{f}$ to $\mathcal{F}_{V}$ is equal to $M_{g}$, where $g=\left.f\right|_{V}$.

(2) $\mathcal{A}_{V}=\left\{\left.f\right|_{V}: f \in \mathcal{A}_{d}\right\}$.

(3) $\mathcal{A}_{d} / I_{V}$ is completely isometrically isomorphic to $\mathcal{A}_{V}$ via the restriction map $\left.f \mapsto f\right|_{V}$ of $\mathcal{A}_{d}$ into $\mathcal{A}_{V}$.

(4) For every $f \in \mathcal{A}_{d}$, $\operatorname{dist}\left(f, I_{V}\right)=\operatorname{dist}\left(f, J_{V}\right)$.

Proof. The first item is just a restatement of Proposition 2.6. By universality of $\mathcal{A}_{d}$, $\mathcal{A}_{V}$ is equal to the compression of $\mathcal{A}_{d}$ to $\mathcal{F}_{V}$. Therefore, by (a slight modification of) Popescu's results [25], $\mathcal{A}_{V}$ is the universal operator algebra generated by a commuting row contraction subject to the relations in $I_{V}=J_{V} \cap \mathcal{A}_{d}$. But so is $\mathcal{A}_{d} / I_{V}$. So these two algebras can be naturally identified. Since compression is restriction, (2) and (3) follow. Item (4) follows from the fact that

$$
\begin{aligned}
\operatorname{dist}\left(f, I_{V}\right) & =\left\|f+I_{V}\right\|_{\mathcal{A} / I_{V}}=\left\|P_{\mathcal{F}_{V}} M_{f} P_{\mathcal{F}_{V}}\right\| \\
& =\left\|f+J_{V}\right\|_{\mathcal{M} / J_{V}}=\operatorname{dist}\left(f, J_{V}\right) .
\end{aligned}
$$

Corollary 7.3. Let $V$ be a homogeneous variety, or a Blaschke sequence in the disc such that $S(V)$ has measure zero. Then $\mathcal{A} / I_{V}$ embeds into $\mathcal{M} / J_{V}$ isometrically.

Define

$$
\bar{V}^{\mathcal{A}}=\left\{\lambda \in \overline{\mathbb{B}_{d}}: f(\lambda)=0 \text { for all } f \in I_{V}\right\} .
$$

Clearly $\bar{V}^{\mathcal{A}}$ contains the closure of $V$ in $\mathbb{B}_{d}$. But it is not clear exactly what else it contains. However, it seems most reasonable to restrict our attention to the algebras 
$\mathcal{A}_{V}$ such that $V=V\left(I_{V}\right)$, so that the variety $V$ is determined by functions in $\mathcal{A}$. In this case, we obtain

$$
\mathbb{B}_{d} \cap \bar{V}^{\mathcal{A}}=V .
$$

The proof is the same as that of Proposition 2.1. It is not clear whether this holds for arbitrary varieties. This equation does hold when $V \subseteq \mathbb{D}$ is a Blaschke sequence and $|S(V)|=0$.

Proposition 7.4. Let $V$ be a variety satisfying condition (7.1). Then the character space $M\left(\mathcal{A}_{V}\right)$ of $\mathcal{A}_{V}$ can be identified with $\bar{V}^{\mathcal{A}}$.

Proof. Let $\lambda \in \bar{V}^{\mathcal{A}}$. Then the evaluation functional $\rho_{\lambda}$ given by $\rho_{\lambda}(f)=f(\lambda)$ is a character of $\mathcal{A}$ with kernel equal to $I_{\{\lambda\}} \supseteq I_{V}$. Thus $\rho_{\lambda}$ can be promoted to a character of $\mathcal{A}_{V}=\mathcal{A} / I_{V}$.

Denote by $Z_{1}, \ldots, Z_{d}$ the images of the coordinate functions in $\mathcal{A}_{V}$. If $\rho$ is a character of $\mathcal{A}_{V}$, let

$$
\lambda=\left(\lambda_{1}, \ldots, \lambda_{d}\right)=\left(\rho\left(Z_{1}\right), \ldots, \rho\left(Z_{d}\right)\right) .
$$

Then $\lambda \in \overline{\mathbb{B}}_{d}$ because $\rho$ is completely contractive. For every $f \in I_{V}, f\left(Z_{1}, \ldots, Z_{d}\right)=$ 0 . Thus

$$
\rho\left(f\left(Z_{1}, \ldots, Z_{d}\right)\right)=f\left(\lambda_{1}, \ldots, \lambda_{d}\right)=0 .
$$

So $\lambda$ lies in the set of all points in $\overline{\mathbb{B}}_{d}$ that annihilate $I_{V}$, which is $\bar{V}^{\mathcal{A}}$.

This identification is easily seen to be a homeomorphism.

Proposition 7.5. Let $V \subseteq \mathbb{B}_{d}$ and $W \subseteq \mathbb{B}_{d^{\prime}}$ be varieties which satisfy condition (7.1). Let $\varphi: \mathcal{A}_{V} \rightarrow \mathcal{A}_{W}$ be a unital algebra homomorphism. Then there exists a holomorphic map $F: \mathbb{B}_{d^{\prime}} \rightarrow \mathbb{C}^{d}$ that extends continuously to $\overline{\mathbb{B}}_{d^{\prime}}$ such that

$$
\left.F\right|_{\bar{W}^{\mathcal{A}}}=\varphi^{*} .
$$

The components of $F$ are in $\mathcal{A}_{d^{\prime}}$, and norm of $F$ as a row of multipliers is less than or equal to the cb-norm of $\varphi$. Moreover, $\varphi$ is given by composition with $F$, that is,

$$
\varphi(f)=f \circ F \quad \text { for } f \in \mathcal{A}_{V} .
$$

Proof. Every character in $M\left(\mathcal{A}_{W}\right)$ is an evaluation functional at some point $\lambda \in$ $\bar{W}^{\mathcal{A}}$. Identifying $\bar{W}^{\mathcal{A}}$ and $M\left(\mathcal{A}_{W}\right)$, we find, as in Lemma 4.2, that the mapping $\varphi^{*}$ is given by

$$
\varphi^{*}(\lambda)=\left(\varphi\left(Z_{1}\right)(\lambda), \ldots, \varphi\left(Z_{d}\right)(\lambda)\right) \quad \text { for all } \lambda \in \bar{W}^{\mathcal{A}} .
$$

Proposition 7.2 implies that $\varphi\left(Z_{1}\right), \ldots, \varphi\left(Z_{d}\right)$ are restrictions to $W$ of functions $f_{1}, \ldots, f_{d}$ in $\mathcal{A}_{d^{\prime}}$. (This is only true under our special assumption (7.1). Otherwise we only get $f_{1}, \ldots, f_{d}$ in $\mathcal{M}_{d^{\prime}}$.) Defining

$$
F(z)=\left(f_{1}(z), \ldots, f_{d}(z)\right)
$$

we obtain the required map $F$. Finally, for every $\lambda \in \bar{W}^{\mathcal{A}}$,

$$
\varphi(f)(\lambda)=\rho_{\lambda}(\varphi(f))=\varphi^{*}\left(\rho_{\lambda}\right)(f)=\rho_{F(\lambda)}(f)=f(F(\lambda)) .
$$

Therefore $\varphi(f)=f \circ F$. 
This immediately yields:

Corollary 7.6. Let $V \subseteq \mathbb{B}_{d}$ and $W \subseteq \mathbb{B}_{d^{\prime}}$ be varieties satisfying condition (7.1). If $\mathcal{A}_{V}$ and $\mathcal{A}_{W}$ are isomorphic, then there are two holomorphic maps $F: \mathbb{B}_{d^{\prime}} \rightarrow \mathbb{C}^{d}$ and $G: \mathbb{B}_{d} \rightarrow \mathbb{C}^{d^{\prime}}$ which extend continuously to the closed balls, such that $F\left(\bar{W}^{\mathcal{A}}\right)=$ $\bar{V}^{\mathcal{A}}, G\left(\bar{V}^{\mathcal{A}}\right)=\bar{W}^{\mathcal{A}}$, and $\left.F\right|_{\bar{W}^{\mathcal{A}}}$ and $\left.G\right|_{\bar{V}^{\mathcal{A}}}$ are inverses of each other. If $V$ and $W$ satisfy condition (7.2), then $F(W)=V$ and $G(V)=W$.

From these results and the techniques of Lemma 4.3, we also get that if $\mathcal{A}_{V}$ and $\mathcal{A}_{W}$ are completely isometrically isomorphic, then there exists an automorphism $F \in \operatorname{Aut}\left(\mathbb{B}_{d}\right)$ such that $F(V)=W$. On the other hand, the completely isometric isomorphisms of Proposition 4.1 are easily seen to respect the norm closures of the polynomials in $\mathcal{M}_{V}$ and $\mathcal{M}_{W}$. Together with the above corollary we obtain the following analogue to Theorem 4.4 .

Theorem 7.7. Let $V$ and $W$ be varieties in $\mathbb{B}_{d}$ satisfying (17.1). Then $\mathcal{A}_{V}$ is completely isometrically isomorphic to $\mathcal{A}_{W}$ if and only if there exists an automorphism $F$ of $\mathbb{B}_{d}$ such that $F(W)=V$.

Every completely isometric isomorphism $\varphi: \mathcal{A}_{V} \rightarrow \mathcal{A}_{W}$ arises as composition $\varphi(f)=f \circ F$, where $F$ is such an automorphism. In this case $\varphi$ is unitarily implemented by a unitary sending the kernel function $k_{w} \in \mathcal{F}_{W}$ to a scalar multiple of the kernel function $k_{F(w)} \in \mathcal{F}_{V}$.

\section{Quotients of $\mathrm{A}(\mathbb{D})$}

Let $V=\left\{z_{n}: n \geq 1\right\}$ be a Blaschke sequence in the disk. Write $B_{V}$ for the Blaschke product with simple zeros at the points in $V$. Observe that $J_{V}=B_{V} H^{\infty}$ and $I_{V}=J_{V} \cap \mathrm{A}(\mathbb{D})$. By Lemma 7.1] and Proposition [7.2, if the measure $|S(V)|$ of $S(V)=\bar{V} \cap \mathbb{T}$ is zero, then $\mathcal{A}_{V}=\left.\mathrm{A}(\mathbb{D})\right|_{V} \cong \mathrm{A}(\mathbb{D}) / I_{V}$.

The interpolating sequences play a special role.

Theorem 8.1. Let $S(V)=\bar{V} \cap \mathbb{T}$.

(1) If $|S(V)|>0$ then $I_{V}=\{0\}$.

(2) If $V$ is interpolating and $|S(V)|=0$, then $\mathcal{A}_{V}$ is isomorphic to $\mathrm{C}(\bar{V})$ by the restriction map.

(3) If $\mathcal{A}_{V}$ is isomorphic to $\mathrm{C}(\bar{V})$ via the restriction map, then $V$ is an interpolating sequence.

Proof. (1) If $|S(V)|>0$, then any $f \in I_{V}$ must vanish on $S(V)$, and hence is 0 .

(2) The map taking $f \in \mathrm{A}(\mathbb{D})$ to $\left.f\right|_{\bar{V}}$ is clearly a contractive homomorphism of $\mathrm{A}(\mathbb{D})$ into $\mathrm{C}(\bar{V})$ with kernel $I_{V}$. So it factors through $\mathcal{A}_{V}$ and induces an injection of $\mathcal{A}_{V}$ into $\mathrm{C}(\bar{V})$. It suffices to show that this map is surjective, for the result then follows from the open mapping theorem.

Fix $h \in \mathrm{C}(\bar{V})$. By Rudin's Theorem (see 22 , p. 81]), there is a function $f \in \mathrm{A}(\mathbb{D})$ such that $\left.f\right|_{S(V)}=\left.h\right|_{S(V)}$. By replacing $h$ with $h-f$, we may suppose that $\left.h\right|_{S(V)}=0$. Hence $\left.h\right|_{V}$ is a function such that $\lim _{n \rightarrow \infty} h\left(z_{n}\right)=0$. Now it suffices to show that if $h\left(z_{n}\right)=0$ for all $n>N$, then there is a function $f \in \mathrm{A}(\mathbb{D})$ with $\left.f\right|_{V}=\left.h\right|_{V}$ and $\|f\| \leq C\|h\|_{\infty}$ for a constant $C$ which is independent of $N$. Surjectivity will follow from a routine approximation argument. Let $c$ be the interpolation constant for $V$. 
Fix $N$. Take $h \in \mathrm{C}(\bar{V})$ with $h\left(z_{n}\right)=0$ for all $n>N$ and $\|h\|_{\infty} \leq 1$. By a theorem of Fatou [22, p. 81], there is an analytic function $g$ on $\mathbb{D}$ such that $\operatorname{Re} g \geq 0$ and $e^{-g} \in \mathrm{A}(\mathbb{D})$ vanishes precisely on $S(V)$. There is an integer $m>0$ so that $\left|e^{-g / m}\left(z_{n}\right)\right|>.5$ for $1 \leq n \leq N$. Set $V_{N}=\left\{z_{n}: n>N\right\}$. Since $V$ is interpolating,

$$
\min \left\{\left|B_{V_{N}}\left(z_{n}\right)\right|: 1 \leq n \leq N\right\} \geq 1 / c .
$$

We will look for a function $f$ of the form $f=B_{V_{N}} e^{-g / m} f_{0}$. By the arguments for Rudin's Theorem, this will lie in $\mathrm{A}(\mathbb{D})$. Clearly it vanishes on $V_{N} \cup S(V)$, and we require

$$
h\left(z_{n}\right)=f\left(z_{n}\right)=B_{V_{N}}\left(z_{n}\right) e^{-g / m}\left(z_{n}\right) f_{0}\left(z_{n}\right) .
$$

So we need to find $f_{0} \in \mathrm{A}(\mathbb{D})$ with $\left\|f_{0}\right\| \leq C$ and

$$
f_{0}\left(z_{n}\right)=a_{n}:=h\left(z_{n}\right) e^{g\left(z_{n}\right) / m} / B_{V_{N}}\left(z_{n}\right) \quad \text { for } 1 \leq n \leq N .
$$

The estimates made show that $\left|a_{n}\right| \leq 2 c$. The interpolation constant for $\left\{z_{n}: 1 \leq\right.$ $n \leq N\}$ is at most $c$, and since this is a finite set, we can interpolate using functions in $\mathrm{A}(\mathbb{D})$ which are arbitrarily close to the optimal norm. Thus we can find an $f_{0}$ with $\left\|f_{0}\right\| \leq 3 c^{2}$. Hence we obtain $f$ with the same norm bound.

(3) If $\mathcal{A}_{V}$ is isomorphic to $\mathrm{C}(\bar{V})$ via the restriction map, then by the open mapping theorem, there is a constant $c$ so that for any $h \in \mathrm{C}(\bar{V})$, there is an $f \in \mathrm{A}(\mathbb{D})$ with $\left.f\right|_{V}=\left.h\right|_{V}$ and $\|f\| \leq c\|h\|$. In particular, for any bounded sequence $\left(a_{n}\right)$ and $N \geq 1$, there is an $f_{N} \in \mathrm{A}(\mathbb{D})$ such that $\left\|f_{N}\right\| \leq c\left\|\left(a_{n}\right)\right\|_{\infty}$ and

$$
f_{N}\left(z_{n}\right)= \begin{cases}a_{n} & \text { if } 1 \leq n \leq N \\ 0 & \text { if } n>N\end{cases}
$$

Take a weak-* cluster point $f$ of this sequence in $H^{\infty}$. Then $\|f\| \leq c$ and $f$ interpolates the sequence $\left(a_{n}\right)$ on $V$. So $V$ is interpolating.

We can now strengthen Example 6.2 showing that there are discrete varieties giving rise to non-isomorphic algebras which are biholomorphic with a biholomorphism that extends continuously to the boundary.

Example 8.2. We will show that there is a Blaschke sequence $V$ which is not interpolating and an interpolating sequence $W$ and functions $f$ and $g$ in $\mathrm{A}(\mathbb{D})$ so that $\left.f\right|_{V}$ is a bijection of $V$ onto $W$ and $\left.g\right|_{W}$ is its inverse. Take

$$
V=\left\{z_{n}:=1-n^{-2}: n \geq 1\right\} \text { and } W=\left\{w_{n}:=1-n^{-2} e^{-n^{2}}: n \geq 1\right\} .
$$

Then $W$ is an interpolating sequence, and $V$ is not. Let

$$
f(z)=1+(z-1) e^{1 /(z-1)} .
$$

Then since $1 /(z-1)$ takes $\mathbb{D}$ conformally onto $\{z: \operatorname{Re} z<-1 / 2\}$, it is easy to see that $e^{1 /(z-1)}$ is bounded and continuous on $\overline{\mathbb{D}} \backslash\{1\}$. Hence $f$ is continuous, so lies in $\mathrm{A}(\mathbb{D})$. Clearly, $f\left(z_{n}\right)=1-n^{-2} e^{-n^{2}}=w_{n}$ for $n \geq 1$. The inverse of $\left.f\right|_{V}$ is the $\operatorname{map} h\left(w_{n}\right)=z_{n}$. Since

$$
\lim _{n \rightarrow \infty} h\left(w_{n}\right)=\lim _{n \rightarrow \infty} z_{n}=1,
$$

this extends to be a continuous function on $\bar{W}=W \cup\{1\}$. By Theorem 8.1, there is a function $g \in \mathrm{A}(\mathbb{D})$ such that $\left.g\right|_{W}=h$. 
Remark 8.3. Let $V=\left\{v_{n}\right\}$ and $W=\left\{w_{n}\right\}$ be two interpolating sequences in $\mathbb{D}$ with $\lim v_{n}=\lim w_{n}=1$. Then the algebras $\mathcal{A}_{V}$ and $\mathcal{A}_{W}$ are both isomorphic to $c$, the space of convergent sequences. As in our counterexamples using Blaschke products, we can find biholomorphisms carrying one sequence onto the other. However, there is no reason for the rates at which they approach the boundary to be comparable.

We now give a strengthening of Theorem 8.1

Theorem 8.4. Let $V=\left\{v_{n}\right\}_{n=1}^{\infty}$ be a Blaschke sequence in $\mathbb{D}$ such that $|S(V)|=0$. Then $\mathcal{A}_{V}$ is isomorphic to $\mathrm{C}(\bar{V})$ if and only if $V$ is interpolating.

Proof. Theorem 8.1 says that $\mathcal{A}_{V}$ is isomorphic to $\mathrm{C}(\bar{V})$ via the restriction map if and only if $V$ is interpolating. All that remains to prove is that if $V$ is not an interpolating sequence, then it cannot be isomorphic via any other isomorphism.

Suppose that $V$ is a non-interpolating sequence and define $w_{n}=\left(1-e^{-n}\right) v_{n} /\left|v_{n}\right|$. Then $W=\left\{w_{n}\right\}$ is an interpolating sequence with $S(W)=S(V)$, and $\bar{V}$ is homeomorphic to $\bar{W}$ via the map that continuously extends $v_{n} \mapsto\left(1-e^{-n}\right) v_{n} /\left|v_{n}\right|$. Therefore, $\mathcal{A}_{W}$ is isomorphic to $\mathrm{C}(\bar{V})$ via the restriction map. Now assume that $\mathcal{A}_{V}$ is isomorphic to $\mathrm{C}(\bar{V})$ by any isomorphism. Then it is isomorphic to $\mathcal{A}_{W}$. But by Corollary 7.6, this isomorphism is given by composition with a holomorphic map. Therefore $\mathcal{A}_{V}$ is isomorphic to $\mathrm{C}(\bar{V})$ via the restriction map-a contradiction.

Remark 8.5. In [16], now improved by [20], we saw that in the case of homogeneous varieties $V$ and $W$, the algebras $\mathcal{A}_{V}$ and $\mathcal{A}_{W}$ are isomorphic if and only if the algebras $\mathcal{M}_{V}$ and $\mathcal{M}_{W}$ are isomorphic. The above discussion shows that this is not true in general. If $V$ and $W$ are two interpolating sequences in $\mathbb{D}$, then $\mathcal{M}_{V}$ and $\mathcal{M}_{W}$ are both isomorphic to $\ell^{\infty}$, whereas the isomorphism classes of $\mathcal{A}_{V}$ and $\mathcal{A}_{W}$ depend on the structure of the limit sets.

\section{ADDED IN PROOF}

In the proof of Lemma 4.2, to conclude that $\varphi^{*}$ takes evaluation functionals to evaluation functionals, one needs to use a result of Kennedy and Yang (arXiv:1302.5191) which shows that $\mathcal{M}_{V}$ has a unique isometric predual (and $\mathcal{M}_{W}$ ). The proof of Theorem 4.4 remains valid when $d=\infty$. One needs to verify that Cartan's uniqueness theorem and Rudin's result that the fixed point set of an automorphism of the ball is affine remain valid.

Corollary [5.4 (3) remains valid for $d=\infty$. One needs again to use the result of Kennedy and Yang on unique preduals to obtain that isometric isomorphisms are weak-* continuous, hence $\rho^{*}$ carries $W$ onto $V$.

A more thorough revision has been posted on arXiv.

\section{REFERENCES}

[1] Jim Agler and John E. McCarthy, Complete Nevanlinna-Pick kernels, J. Funct. Anal. 175 (2000), no. 1, 111-124, DOI 10.1006/jfan.2000.3599. MR.1774853 (2001h:47019)

[2] Jim Agler and John E. McCarthy, Pick interpolation and Hilbert function spaces, Graduate Studies in Mathematics, vol. 44, American Mathematical Society, Providence, RI, 2002. MR:1882259(2003b:47001)

[3] Daniel Alpay, Mihai Putinar, and Victor Vinnikov, A Hilbert space approach to bounded analytic extension in the ball, Commun. Pure Appl. Anal. 2 (2003), no. 2, 139-145, DOI 10.3934/cpaa.2003.2.139. MR.1975056(2004b:47013) 
[4] N. Arcozzi, R. Rochberg, and E. Sawyer, Carleson measures for the Drury-Arveson Hardy space and other Besov-Sobolev spaces on complex balls, Adv. Math. 218 (2008), no. 4, 11071180, DOI 10.1016/j.aim.2008.03.001. MR.2419381 (2009j:46062)

[5] Alvaro Arias and Frédéric Latrémolière, Ergodic actions of convergent Fuchsian groups on quotients of the noncommutative Hardy algebras, Proc. Amer. Math. Soc. 139 (2011), no. 7, 2485-2496, DOI 10.1090/S0002-9939-2011-10794-9. MR.2784814

[6] Alvaro Arias and Gelu Popescu, Factorization and reflexivity on Fock spaces, Integral Equations Operator Theory 23 (1995), no. 3, 268-286, DOI 10.1007/BF01198485. MR.1356335 (97e:47066)

[7] Alvaro Arias and Gelu Popescu, Noncommutative interpolation and Poisson transforms, Israel J. Math. 115 (2000), 205-234, DOI 10.1007/BF02810587. MR.1749679 (2001i:47021)

[8] William Arveson, Subalgebras of $C^{*}$-algebras. III. Multivariable operator theory, Acta Math. 181 (1998), no. 2, 159-228, DOI 10.1007/BF02392585. MR.1668582 (2000e:47013)

[9] Frank F. Bonsall and John Duncan, Complete normed algebras, Springer-Verlag, New York, 1973. Ergebnisse der Mathematik und ihrer Grenzgebiete, Band 80. MR0423029(54 \#11013)

[10] S. Costea, E. Sawyer and B. Wick, The Corona Theorem for the Drury-Arveson Hardy space and other holomorphic Besov-Sobolev spaces on the unit ball in $\mathbb{C}^{n}$, Anal. PDE 4 (2011), 499-550. MR3077143

[11] H. G. Dales, Automatic continuity: a survey, Bull. London Math. Soc. 10 (1978), no. 2, 129-183, DOI 10.1112/blms/10.2.129. MR500923(80c:46053)

[12] Kenneth R. Davidson and Ryan Hamilton, Nevanlinna-Pick interpolation and factorization of linear functionals, Integral Equations Operator Theory 70 (2011), no. 1, 125-149, DOI 10.1007/s00020-011-1862-7. MR2786738(2012e:47045)

[13] Kenneth R. Davidson and David R. Pitts, Invariant subspaces and hyper-reflexivity for free semigroup algebras, Proc. London Math. Soc. (3) 78 (1999), no. 2, 401-430, DOI 10.1112/S002461159900180X. MR.1665248 (2000k:47005)

[14] Kenneth R. Davidson and David R. Pitts, The algebraic structure of non-commutative analytic Toeplitz algebras, Math. Ann. 311 (1998), no. 2, 275-303, DOI 10.1007/s002080050188. MR.1625750 (2001c:47082)

[15] Kenneth R. Davidson and David R. Pitts, Nevanlinna-Pick interpolation for noncommutative analytic Toeplitz algebras, Integral Equations Operator Theory 31 (1998), no. 3, 321-337, DOI 10.1007/BF01195123. MR.1627901 (2000g:47016)

[16] Kenneth R. Davidson, Christopher Ramsey, and Orr Moshe Shalit, The isomorphism problem for some universal operator algebras, Adv. Math. 228 (2011), no. 1, 167-218, DOI 10.1016/j.aim.2011.05.015. MR.2822231(2012j:46083)

[17] John B. Garnett, Bounded analytic functions, Pure and Applied Mathematics, vol. 96, Academic Press Inc. [Harcourt Brace Jovanovich Publishers], New York, 1981. MR628971 (83g:30037)

[18] Robert C. Gunning, Introduction to holomorphic functions of several variables. Vol. II, The Wadsworth \& Brooks/Cole Mathematics Series, Wadsworth \& Brooks/Cole Advanced Books \& Software, Monterey, CA, 1990. Local theory. MR.1057177 (92b:32001b)

[19] Robert C. Gunning and Hugo Rossi, Analytic functions of several complex variables, PrenticeHall Inc., Englewood Cliffs, N.J., 1965. MR0180696 (31 \#4927)

[20] Michael Hartz, Topological isomorphisms for some universal operator algebras, J. Funct. Anal. 263 (2012), no. 11, 3564-3587, DOI 10.1016/j.jfa.2012.08.028. MR2984075

[21] Kenneth Hoffman, Bounded analytic functions and Gleason parts, Ann. of Math. (2) 86 (1967), 74-111. MR0215102 (35 \#5945)

[22] Kenneth Hoffman, Banach spaces of analytic functions, Prentice-Hall Series in Modern Analysis, Prentice-Hall Inc., Englewood Cliffs, N. J., 1962. MR0133008 (24 \#A2844)

[23] Michael T. Jury, Reproducing kernels, de Branges-Rovnyak spaces, and norms of weighted composition operators, Proc. Amer. Math. Soc. 135 (2007), no. 11, 3669-3675 (electronic), DOI 10.1090/S0002-9939-07-08931-9. MR2336583 (2008h:47050)

[24] Gelu Popescu, Poisson transforms on some $C^{*}$-algebras generated by isometries, J. Funct. Anal. 161 (1999), no. 1, 27-61, DOI 10.1006/jfan.1998.3346. MR.1670202 (2000m:46117)

[25] Gelu Popescu, Operator theory on noncommutative varieties, Indiana Univ. Math. J. 55 (2006), no. 2, 389-442, DOI 10.1512/iumj.2006.55.2771. MR2225440(2007m:47008)

[26] Gelu Popescu, Free holomorphic automorphisms of the unit ball of $B(\mathcal{H})^{n}$, J. Reine Angew. Math. 638 (2010), 119-168, DOI 10.1515/CRELLE.2010.005. MR2595338 (2012c:46161) 
[27] Walter Rudin, Function theory in the unit ball of $\mathbf{C}^{n}$, Grundlehren der Mathematischen Wissenschaften [Fundamental Principles of Mathematical Science], vol. 241, Springer-Verlag, New York, 1980. MR601594 (82i:32002)

[28] Hassler Whitney, Complex analytic varieties, Addison-Wesley Publishing Co., Reading, Mass.-London-Don Mills, Ont., 1972. MR.0387634 (52 \#8473)

Department of Pure Mathematics, University of Waterloo, Waterloo, Ontario, CANADA N2L 3G1

E-mail address: krdavids@math.uwaterloo.ca

Department of Pure Mathematics, University of Waterloo, Waterloo, Ontario, CANADA N2L 3G1

E-mail address: ciramsey@math.uwaterloo.ca

Current address: Department of Mathematics, University of Virginia, Charlottesville, Virginia 22904-4137

E-mail address: chramsey@gmail.com

Department of Mathematics, Ben-Gurion University of the Negev, 84105 Be'er Sheva, ISRAEL

E-mail address: oshalit@math.bgu.ac.il 